Check for updates

Cite this: J. Mater. Chem. C, 2020, 8, 5600

Received 13th February 2020 Accepted 20th March 2020

DOI: $10.1039 / \mathrm{d0tc00749h}$

rsc.li/materials-c

\section{Bright and efficient red emitting electroluminescent devices fabricated from ternary europium complexes $\dagger$}

\author{
Muhammad S. Khan, (D)*a Rashid IImi, (D)*a Weidong Sun, José D. L. Dutra, \\ Willyan F. Oliveira, ${ }^{C}$ Liang Zhou, (D) *b Wai-Yeung Wong (iD *d and \\ Paul R. Raithby iD *e
}

\begin{abstract}
The photophysical properties of two previously synthesized, highly efficient, bright red emitting complexes [Eu(btfa) ${ }_{3}$ DPEPO] (CIE, 0.672; 0.326) (Eu-1) and [Eu(nta) $\left.)_{3} \mathrm{DPEPO}\right](\mathrm{ClE}, 0.673 ; 0.326)(\mathrm{Eu}-2)$ (btfa = 4,4,4-trifluoro-1-phenyl-1,3-butanedione, nta = 4,4,4-trifluoro-1-(2-naphthyl)-1,3-butanedione and DPEPO = bis(2-(diphenylphosphino)phenyl)ether oxide) are reported. The complexes have been used as dopants to fabricate several single and double emitting layer (EML) electroluminescent (EL) devices. At the optimum doping concentration of $4 \mathrm{wt} \%$, the single EML device of Eu-1 exhibited a pure red color with an external quantum efficiency (EQE) of $2.9 \%$ and a maximum brightness $(B)=1320 \mathrm{~cd} \mathrm{~m}^{-2}$, maximum current efficiency $\left(\eta_{\mathrm{c}}\right)=4.08 \mathrm{~cd} \mathrm{~A}^{-1}$, maximum power efficiency $\left(\eta_{\mathrm{p}}\right)=3.37 \mathrm{~lm} \mathrm{~W}^{-1}$ with a very low turn-on voltage $\left(V_{\text {turn-on }}\right)=3.8 \mathrm{~V}$. Importantly, the single EML device of Eu-2 at the optimum doping concentration exhibited pure red EL with EQE of $6.0 \%$ with the best EL performance: $B=2108 \mathrm{~cd} \mathrm{~m}^{-2}, \eta_{\mathrm{c}}=$ $8.45 \mathrm{~cd} \mathrm{~A}^{-1}$, and $\eta_{\mathrm{p}}=6.98 \mathrm{~lm} \mathrm{~W}^{-1}$ at a very low $V_{\text {turn-on }}=3.5 \mathrm{~V}$. The performances of this device are among the best reported for devices incorporating a europium complex as a red emitter. Furthermore, the EL performances were achieved at the current density of $10 \mathrm{~mA} \mathrm{~cm}{ }^{-2}$, thereby alleviating a serious efficiency rolloff as a major obstacle to the development of Eu-complex based EL devices.
\end{abstract}

\section{Introduction}

Efficient photoluminescent materials with desirable properties are becoming increasingly crucial for the development of organic light-emitting devices (OLEDs), luminescence thermometer (LMT), bioassay, etc. ${ }^{1}$ A plethora of reports on photoluminescent materials comprising either organic or metal complexes have appeared and many of the materials have fascinating applications. Among the materials in this category trivalent lanthanide [Ln(III)] complexes, especially Eu(III) coordination complexes (Eu-CCs) have the shown potential to be used in a range of

\footnotetext{
${ }^{a}$ Department of Chemistry, Sultan Qaboos University, P. O. Box 36, Al Khod 123, Muscat, Oman.E-mail: rashidilmi@gmail.com, msk@squ.edu.om

${ }^{b}$ State Key Laboratory of Rare Earth Resource Utilization, Changchun Institute of Applied Chemistry, Chinese Academy of Sciences, Renmin Street 5625,

Changchun 130022, People's Republic of China. E-mail: zhoul@ciac.ac.cn

${ }^{c}$ Pople Computational Chemistry Laboratory, Department of Chemistry, UFS, 49100-000 São Cristóvão, Sergipe, Brazil

${ }^{d}$ Department of Applied Biology and Chemical Technology, The Hong Kong Polytechnic University, Hung Hom, Kowloon, Hong Kong, People's Republic of China. E-mail: wai-yeung.wong@polyu.edu.hk

${ }^{e}$ Department of Chemistry, University of Bath, Claverton Down, Bath BA2 7AY, UK E-mail:p.r.raithby@bath.ac.uk

$\dagger$ Electronic supplementary information (ESI) available. See DOI: 10.1039/d0tc00749h
}

applications such as OLEDs, ${ }^{2}$ sensors, ${ }^{3}$ sensitizers to improve the electroluminescence (EL) performance of red-emitting $\operatorname{Ir}$ (III) complex, ${ }^{1 a, 4}$ and laser materials. ${ }^{5}$ This is because of their intriguing photophysical properties, e.g., highly monochromatic red emission (full width at half maxima (FWHM) less than $\left.10 \mathrm{~nm}, \mathrm{CIE}_{x, y}=0.67,0.32\right),{ }^{5 b, c, 6}$ high photoluminescence quantum yield (PLQY), ${ }^{7}$ large energy shift between absorbed and emitted radiation, millisecond ( $\mathrm{ms}$ ) to microsecond $(\mu \mathrm{s})$ long luminescence lifetimes. However, according to the Laporte rule ${ }^{8}$ for the optical absorption, the redistribution of electrons within a given subshell is forbidden. This limitation can be overcome by the introduction of appropriate organic chromophore(s) (referred to as "antenna" ligands) with high absorption coefficients $(\varepsilon)$ in ultraviolet (UV) or visible (Vis) region of the spectrum to harvest the absorbed energy to the Ln(III) emitting state, typically known as the "antenna effect".

Among the many strongly absorbing chelating multidentate organic antenna ligands, acetylacetone (Hacac) and its derivatives, known as $\beta$-diketones ( $\beta$-diket) have dominated lanthanide coordination chemistry. ${ }^{10}$ This is primarily because of their ease of synthesis, high $\varepsilon$ values, and intense red or green emission, which is caused by the high energy transfer (ET) from the $\beta$-diket to the $\mathrm{Eu}(\mathrm{III})$ or $\mathrm{Tb}$ (III) ion emitting states. Most importantly, 
they form strongly coordinated neutral and stable lanthanide coordination complexes (Ln-CCs). However, water or alcohol molecule(s) always complement the unsaturated coordination sphere affecting the overall photophysical properties due to the vibronic quenching by high energy oscillators. This issue can be simply resolved by utilizing co-ligands. One of the co-ligands of choice is rigid "bis(2-(diphenylphosphino)phenyl)ether oxide (DPEPO)" because of its intriguing properties. It serves as a hard Lewis base forming very stable complexes with hard Lewis acid [Ln(III)] ions and as an excellent photosensitizer for the Ln(III) ions generating high PLQY (up to 80\%) with $97 \%$ sensitization efficiency $\left(\eta_{\text {sen }}\right){ }^{7 a}$ It provides a large bite angle that imposes a distortion to the complex symmetry, leading to additional enhancement of PLQY. Furthermore, the coordination of DPEPO increases the thermal- and photostability of the complexes, thus complexes formed would be ideal candidates for the fabrication of OLEDs via a thermal evaporation process. An additional advantage of DPEPO lies in its capacity to be used as a host material for the EML in OLEDs. ${ }^{2 a, 11}$ In continuation of our on-going research to improve the EL performance of the redemitting Eu(III) complexes, herein, we report systematic and comparative photophysical analyses of two complexes [Eu(btfa $\left.)_{3} \mathrm{DPEPO}\right]$ (CIE, 0.672; 0.326) (Eu-1) and [Eu(nta) $)_{3}$ DPEPO] (CIE, 0.673; 0.326) (Eu-2) (Chart 1) first reported by Hasegawa et al. ${ }^{12}$ Our experimental and theoretical studies include determination of Judd-Ofelt $(\mathrm{J}-\mathrm{O})$ intensity parameter $\left(\Omega_{2}\right.$ and $\left.\Omega_{4}\right)$, radiative $\left(A_{\mathrm{R}}\right)$ and non-radiative $\left(A_{\mathrm{NR}}\right)$ rate constants, radiative lifetime $\left(\tau_{\mathrm{rad}}\right)$, forward $\left(W_{\mathrm{ET}}\right)$ and backward $\left(W_{\mathrm{BT}}\right)$ ET rates and intrinsic quantum yield $\left(Q_{\mathrm{Eu}}^{\mathrm{Eu}}\right)$. Since the pioneering works of Kido et al., ${ }^{13}$ on the EL of lanthanide complexes, research on OLEDs is growing because of their potential use in the next-generation flat panel displays and solid-state lighting (SSL) applications. Xu et al., ${ }^{14}$ used bidentate DPEPO ligand to synthesize a $\left[\mathrm{Eu}(\mathrm{tta})_{3} \mathrm{DPEPO}\right]$ complex $(\mathrm{tta}=2$-thenoyltrifluoroacetonate) and utilized it to fabricate red OLEDs. The fabricated device displayed impressive brightness $(B)$ of $632 \mathrm{~cd} \mathrm{~m}^{-2}$ at $25 \mathrm{~V}$ with maximum EQE of $2.89 \%$ and $\eta_{\mathrm{c}}$ and $\eta_{\mathrm{p}}$ of $4.58 \mathrm{~cd} \mathrm{~A}^{-1}$ and $2.05 \mathrm{~lm} \mathrm{~W}^{-1}$ were achieved at $7 \mathrm{~V}$. Later, the same group reported $^{15} \mathrm{EL}$ devices of modified monodentate phosphine oxide ligands and synthesized $\left[\mathrm{Eu}(\mathrm{tta})_{3}(\mathrm{tapo})_{2}\right],\left[\mathrm{Eu}(\mathrm{tta})_{3}(\text { nadapo })_{2}\right]$ and $\left[\mathrm{Eu}(\mathrm{tta})_{3}(\mathrm{cppo})_{2}\right]$ (tapo $=$ 4-diphe-nylaminophenyl)diphenylphosphineoxide, (nadapo = naphthalen-1-yl-phe-nylaminophenyl)diphenylphosphineoxide and cppo $=[4-(($ diphenyl-phosphinoyl $)-$ phenyl]-9H-carbazole). Inspiring EL performances were obtained from the devices using these complexes as an EML with $B=1195 \mathrm{~cd} \mathrm{~m}^{-2}$ at $19.6 \mathrm{~V}$ and $\mathrm{EQE}=3.2 \%$, for $\left[\mathrm{Eu}(\mathrm{tta})_{3}(\operatorname{tapo})_{2}\right]$, $B=1195 \mathrm{~cd} \mathrm{~m}^{-2}$ at $18.0 \mathrm{~V}$ and $\mathrm{EQE}=3.71 \%$ for $\left[\mathrm{Eu}(\mathrm{tta})_{3}(\text { nadapo })_{2}\right]$ and $B=852 \mathrm{~cd} \mathrm{~m}^{-2}$ at $22.2 \mathrm{~V}$ and $\mathrm{EQE}=2.08 \%$, respectively. However, these devices utilized very high operating voltages to achieve these EL performances and are therefore not suitable for real-life applications. The objective of producing low $V_{\text {turn-on, }}$, Eu-CCs based red-emitting OLEDs is important and needs to be investigated further. Based on this idea, Zhao et al. ${ }^{16}$ utilized exciplex of $4,4^{\prime}, 4^{\prime \prime}$-tri( $N$-carbazolyl)-triphenylamine:4,7-diphenyl1,10-phenanthroline (TCTA:Bphen) as a host to sensitize the $\left[\mathrm{Eu}(\mathrm{DBM})_{3}\right.$ Phen] complex to achieve a high purity red color OLED. At the optimum doping concentration of $10 \mathrm{wt} \%$, the device exhibited $\eta_{\mathrm{c}}$ and EQE of $3.85 \mathrm{~cd} \mathrm{~A}^{-1}$ and $2.12 \%$ at $V_{\text {turn-on }}=$ $2.9 \mathrm{~V}$. Recently, we have reported a light red $\left(\mathrm{CIE}_{x, y}, 0.574 ; 0.275\right)$ EL device of $\left[\mathrm{Eu}(\mathrm{tfac})_{3} \mathrm{DPEPO}\right]{ }^{2 a}$ (tfac = trifluoroacetylacetonate) with an impressive $B=1274 \mathrm{~cd} \mathrm{~m}^{-2}$ at very low $V_{\text {turn-on }}=3.4 \mathrm{~V}$.

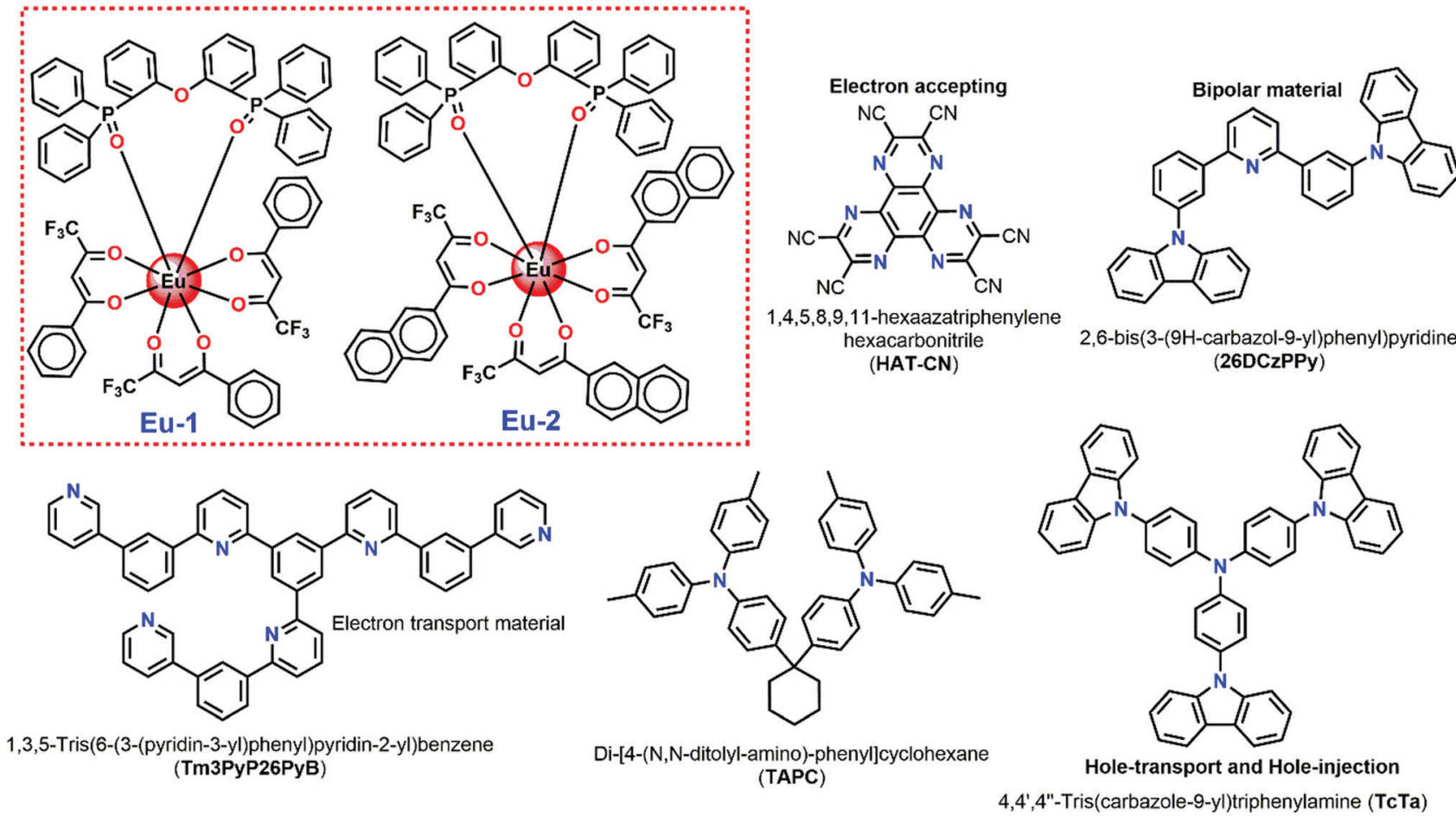

Chart 1 Structural representation of Eu complexes and chemicals used in EL device fabrication. 
Motivated by these findings, we further employed the Eu-1 and Eu-2 complexes as EML to fabricate single- and double-layer OLEDs. We found that at the optimum doping concentration of $4 \mathrm{wt} \%$ Eu-2, single-layer devices outperformed many of the Eu-CCs and displayed the best EL performances, $B, \eta_{\mathrm{c}}, \eta_{\mathrm{p}}$, and

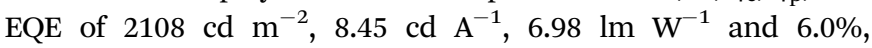
respectively, at very low $V_{\text {turn-on }}=3.4 \mathrm{~V}$.

\section{Photophysical parameters}

Details of the syntheses and photophysical measurements of Eu-1 and Eu-2 (Chart 1) are given in the ESI. $\dagger$ Important experimental photophysical parameters such as J-O parameters $\left(\Omega_{2}\right.$ and $\left.\Omega_{4}\right)$, radiative $\left(A_{\mathrm{R}}\right)$ and non-radiative $\left(A_{\mathrm{NR}}\right)$ decay rates, radiative lifetime $\left(\tau_{\mathrm{rad}}\right)$ and intrinsic quantum yield $\left(Q_{\mathrm{Eu}}^{\mathrm{Eu}}\right)$ were calculated using following eqn (1)-(7) and details are reported elsewhere. $^{1 a, 17}$

$$
\begin{gathered}
\Omega_{\lambda}^{\exp }=\frac{3 \hbar A_{\mathrm{R}}\left[{ }^{5} \mathrm{D}_{0} \rightarrow{ }^{7} \mathrm{~F}_{J}\right]}{32 e^{2} \pi^{3} \chi v\left[{ }^{5} \mathrm{D}_{0} \rightarrow{ }^{7} \mathrm{~F}_{J}\right]{ }^{3}\left|\left\langle{ }^{5} \mathrm{D}_{0}\|U(\lambda)\|{ }^{7} \mathrm{~F}_{J}\right\rangle\right|^{2}} \\
A_{\mathrm{R}}=\sum_{J=0}^{4} A_{\mathrm{R}}\left[{ }^{5} \mathrm{D}_{0} \rightarrow{ }^{7} \mathrm{~F}_{J}\right] \\
A_{\mathrm{R}}\left[{ }^{5} \mathrm{D}_{0} \rightarrow{ }^{7} \mathrm{~F}_{J}\right]=\frac{v\left[{ }^{5} \mathrm{D}_{0} \rightarrow{ }^{7} \mathrm{~F}_{1}\right]}{v\left[{ }^{5} \mathrm{D}_{0} \rightarrow{ }^{7} \mathrm{~F}_{J}\right]} \times \frac{A\left[{ }^{5} \mathrm{D}_{0} \rightarrow{ }^{7} \mathrm{~F}_{J}\right]}{A\left[{ }^{5} \mathrm{D}_{0} \rightarrow{ }^{7} \mathrm{~F}_{1}\right]} A_{\mathrm{R}}\left[{ }^{5} \mathrm{D}_{0} \rightarrow{ }^{7} \mathrm{~F}_{1}\right] \\
A_{\mathrm{tot}}=\frac{1}{\tau_{\mathrm{obs}}}=A_{\mathrm{R}}+A_{\mathrm{NR}} \\
Q_{\mathrm{Eu}}^{\mathrm{Eu}}=\frac{\tau_{\mathrm{obs}}}{\tau_{\mathrm{rad}}}=\frac{\tau_{\mathrm{rad}}}{A_{\mathrm{R}}+A_{\mathrm{NR}}} \\
\eta_{\mathrm{sen}}=\frac{Q_{\mathrm{Eu}}^{\mathrm{L}}}{Q_{\mathrm{Eu}}^{\mathrm{Eu}}}
\end{gathered}
$$

\subsection{Details of theoretical calculations}

2.1.1. Ground state geometry and singlet (S) and triplet (T) energy levels. Structural modeling of Eu-1 and Eu-2 was performed using a reference crystal structure of an analogous europium complex (CSD CODE 1942486) reported by us. ${ }^{2 a}$ The structures were optimized without any symmetry restrictions using the RM1 model ${ }^{18}$ implemented in the MOPAC2016 program. ${ }^{19}$ A search in the Cambridge Crystallographic Database $(\mathrm{CSD})^{20}$ for $\left[\mathrm{Ln}(\beta \text {-diket })_{3} \mathrm{DPEPO}\right]$ complexes showed that $\beta$-diketonate ligands with different substituents adopt different relative positions with respect to the orientation of the same substituent. As a result, eight different starting structures were considered for Eu-1 and Eu-2. Fig. S1 and S2, ESI $\dagger$ show the geometries optimized with RM1 for all structures considered for both complexes. Vibrational frequency calculations using the RM1 model showed that all the optimized structures for both complexes were at a minimum of energy.

Additionally, density functional theory (DFT) calculations with the B3LYP/SVP level of theory, implemented into ORCA 4.2.1, ${ }^{21}$ were also performed by using as a starting structure with the lowest energy RM1 geometry for both complexes. The SVP basis function treats the electronic structure of hydrogen $(\mathrm{H})$ atom as $(4 \mathrm{~s} 1 \mathrm{p}) /[2 \mathrm{~s} 1 \mathrm{p}]$, carbon $(\mathrm{C})$, oxygen $(\mathrm{O})$ and fluorine $(\mathrm{F})$ atoms as $(7 \mathrm{~s} 4 \mathrm{p} 1 \mathrm{~d}) /[3 \mathrm{~s} 2 \mathrm{p} 1 \mathrm{~d}]$, and phosphorus $(\mathrm{P})$ as $(10 \mathrm{~s} 7 \mathrm{p} 1 \mathrm{~d}) /$ [4s3p1d]. The electronic structure of Eu was represented by the MWB52 effective core potential, ${ }^{22}$ which represents the core by $46+4 \mathrm{f}^{6}$, with the valence orbitals being treated as $(7 \mathrm{~s} 6 \mathrm{p} 5 \mathrm{~d}) /$ [5s4p3d]. The advantage of using a small optimized basis set is that it reduces the computational cost compared to a calculation involving all electrons. Since Eu-1 and Eu-2 have 133 and 151 atoms, respectively, the use of a basis set containing even more components is prohibitive, so the cost-effective SVP is an appropriate basis set. ${ }^{23}$

Singlet and triplet states of the coordinated ligands were determined from the B3LYP/SVP/MWB52 geometries. Therefore, the Time Dependent-Density Functional Theory (TD-DFT) approach to the CAM-B3LYP/SVP/MWB52 level of theory implemented in ORCA4.2.1 was applied. The effect of the dichloromethane (DCM) solvent was implicitly considered in the excited state calculations using the Conductor-like Polarizable Continuum Model approximation (CPCM). In addition to the TD-DFT approach, INDO/S-CIS semiempirical model, in which the $\mathrm{Ln}$ (III) ion is replaced by a $3 e^{+}$ point charge, has also been applied for comparative purposes. For the INDO configuration interaction single (CIS) excitation approach, 20 occupied molecular orbitals (MOs) and 20 virtual MOs were considered. The absorption spectra were fitted to a Lorentzian function with a half-width at half maximum (HWHM) of $15 \mathrm{~nm}$ for all calculations. Details of the theoretical J-O intensity parameters $\left(\Omega_{\lambda}, \lambda=2,4\right.$ and 6) and eqn (S1)-(S9) are included in ESI. $\dagger$

2.1.2. Energy transfer (ET) rates. According to Malta's model, ${ }^{24}$ if the direct coulombic interaction is only taken into account, the ligand-metal ET rate is calculated by:

$$
W_{\mathrm{ET}}^{\mathrm{C}}=\frac{2 \pi}{\hbar} \frac{e^{2} S_{\mathrm{L}} F}{G(2 J+1)} \sum_{\lambda=2,4,6} \Lambda_{\lambda}\left\langle\psi^{\prime} J^{\prime}\left\|U^{(\lambda)}\right\| \psi J\right\rangle^{2}
$$

$$
\begin{aligned}
\Lambda_{\lambda}= & 2 \Omega_{\lambda}^{\mathrm{FED}}\left(1-\sigma_{1}\right)^{2}\left(\frac{1}{R_{\mathrm{L}}{ }^{6}}\right) \\
& +\left\langle r^{\lambda}\right\rangle^{2}\left\langle 3\left\|C^{(\lambda)}\right\| 3\right\rangle^{2}\left(1-\sigma_{\lambda}\right)^{2}\left(\frac{\lambda+1}{\left(R_{L}^{\lambda+2}\right)^{2}}\right)
\end{aligned}
$$

The reduced matrix element $\left\langle\Psi^{\prime} J^{\prime}\left\|U^{(\ell)}\right\| \Psi J\right\rangle^{2}$ provides the selection rule for total angular momentum $J$ and is given by $|\Delta J| \leq 6$ with $|\Delta J|=2,4,6\left(J=J^{\prime}=0\right.$ excluded $)$. As a result, this mechanism is operative to promote the electronic excitation, for instance, involving the states ${ }^{5} \mathrm{D}_{4} \leftarrow{ }^{7} \mathrm{~F}_{0},{ }^{5} \mathrm{G}_{6} \leftarrow{ }^{7} \mathrm{~F}_{0}$, and ${ }^{5} \mathrm{~L}_{6} \leftarrow{ }^{7} \mathrm{~F}_{0}$ for the $\mathrm{Eu}(\mathrm{III})$ ion. The values of $\left\langle\alpha^{\prime} J^{\prime}\left\|U^{(\ell)}\right\| \alpha J\right\rangle^{2}$ is taken from the work of Carnall et al., ${ }^{25}$ and are implemented into LUMPAC $^{26}$ and considered for calculation of ET rates for Eu-1 
and Eu-2. The ref. 27 describes all quantities of the eqn (8) and (9) in detail.

The contribution regarding the exchange (Ex) mechanism for the ligand-metal ET rate is given by:

$$
\begin{aligned}
W_{\mathrm{ET}}^{\mathrm{Ex}}= & \frac{8 \pi}{3 \hbar} \frac{e^{2}}{R_{\mathrm{L}}^{4}} \frac{\left(1-\sigma_{0}\right)^{2} F}{G(2 J+1)}\left\langle\psi^{\prime} J^{\prime}\|S\| \psi J\right\rangle^{2} \\
& \times \sum_{m}\left\langle\Psi_{N-1} \Pi\left|\sum_{j} r_{j} C_{0}^{(1)}(j) s_{-m}(j)\right| \Psi_{N-1} \Pi^{*}\right\rangle 2
\end{aligned}
$$

From the matrix elements $\left\langle\psi^{\prime} J^{\prime}\|S\| \psi J\right\rangle^{2}$ the selection rule is derived for the Ex mechanism (ExM), which is given by $|\Delta J|=0$, $\pm 1\left(J=J^{\prime}=0\right.$ excluded) and $\Delta S=0$ for the $\operatorname{Ln}$ (III) ion, the latter can be relaxed by the spin-orbit coupling. In this way, the ExM is operative for the excitations ${ }^{5} \mathrm{D}_{1} \leftarrow{ }^{7} \mathrm{~F}_{0}$ and ${ }^{5} \mathrm{D}_{0} \leftarrow{ }^{7} \mathrm{~F}_{1}$ for the $\mathrm{Eu}(\mathrm{III})$ ion. For more details of the terms in the eqn (10) ref. 27 should be consulted.

The calculation of the factor $F$ present in eqn (8) and (10) can be approximated by the following expression:

$$
F=\frac{1}{\hbar \gamma_{\mathrm{L}}} \sqrt{\frac{\ln 2}{\pi}} \exp \left[-\left(\frac{\Delta}{\hbar \gamma_{\mathrm{L}}}\right)^{2} \ln 2\right]
$$

The expression assumes that ligand bandwidth at half-height, $\gamma_{\mathrm{L}}\left(\right.$ in $\mathrm{s}^{-1}$ ), is much larger than the widths of the $4 \mathrm{f}-4 \mathrm{f}$ transitions $\gamma_{\text {Ln }}$ of $\operatorname{Ln}($ III) ions. $\Delta$ is the energy difference between the donor (excited singlet and triplet states of the organic part of the complex in question and acceptor excited states of the lanthanide ion) involved in the ET process.

The term $\left(1-\sigma_{0}\right)$ in eqn (10), recently revisited ${ }^{27}$ by the group led by Malta is given by eqn (12). Utilizing eqn (12) the new version of the equation for calculating ET rate via ExM is formed and implemented in LUMPAC software ${ }^{26}$

$$
\begin{gathered}
\left(1-\sigma_{0}\right)=\rho\left(\frac{R_{\min }}{R_{\mathrm{L}}}\right)^{7 / 2} \\
R_{\mathrm{L}}=\frac{\sum_{i} c_{i}^{2} R_{\mathrm{L}_{i}}}{\sum_{i} c_{l}^{2}}
\end{gathered}
$$

where $\rho$ is the overlap integral (ca. 0.05) between the valence shells of the $\operatorname{Ln}(\mathrm{III})$ ion and the ligating atom and $R_{\min }$ is the smallest distance in the first coordination sphere. $R_{\mathrm{L}}$ is the distance from the $\mathrm{Ln}$ (III) ion nucleus to the electronic barycenter of the ligand donor (or acceptor) state, $c_{i}$ is the molecular orbital coefficient of atom- $i$ at the ligand donor (or acceptor) state and $R_{\mathrm{L}_{i}}$ is the distance of atom- $i$ to the $\operatorname{Ln}(\mathrm{III})$ ion.

Back ET rate is obtained simply by multiplying the direct ET rate by the Boltzmann factor $\exp \left(\frac{-|\Delta|}{k_{\mathrm{B}} T}\right)$, where $T$ is the environment temperature and $k_{\mathrm{B}}$ stands for the Boltzmann constant. The theoretical quantum yields of the complexes were also determined and the details are presented in the ESI $\dagger$ (eqn (S10) and (S11)).

\subsection{Fabrication of EL devices}

The molecular structure of the materials used in the fabrication processes, together with the complexes Eu-1 and Eu-2 are shown in Chart 1. All the organic materials used in the fabrication processes were obtained commercially and used as received without further purification. The device fabrication details are given in the ESI. $\dagger$

\section{Results and discussion}

\subsection{Synthesis and ground state geometry of the europium complexes}

Mixed ligand europium complexes Eu-1 and Eu-2 were synthesized as previously reported ${ }^{12}$ and were characterized by the elemental analysis, mass spectrometry and FTIR spectroscopy (Fig. S3-S6, ESI $\dagger$ ) which are in agreement with the proposed chemical structures and stoichiometry, as shown in Chart 1. Repeated attempts to obtain single crystals of the complexes failed to produce suitable single crystals for X-ray analysis. As a matter of fact, for predicting the photophysical properties of the complex, one needs to have the theoretical structure of the complex, even though the experimental X-ray structure is available. In view of this, the structure of both Eu-1 and Eu-2 were first optimized at the semi-empirical and DFT levels. The root-mean-square-deviation (RMSD) between the geometries optimized by RM1 and B3LYP/SVP/MWB52 are $0.9882 \AA$ and $0.5340 \AA$ for the Eu-1 and Eu-2 complexes, respectively (Fig. S7, ESI $\dagger)$. Although the different methods describe the ligand orientation slightly differently for both complexes, the first coordination sphere remains in good agreement for both the methods. Fig. 1 shows the geometry of Eu-1 and Eu-2 optimized with B3LYP/SVP/MWB52. The information on the polyhedral point groups for the complexes was obtained from a brute force symmetry analyzer implemented into $\mathrm{C}++$ code (https://github. com/nquesada/symmetry). The coordination polyhedron of the B3LYP/SVP/MWB52 model for Eu-1 and Eu-2 (Table S1, ESI $\dagger$ ) showed a low symmetry $C_{1}$ point group. Even by flexing specific parameters of the code, the $C_{1}$ symmetry remains unaltered. The coordination geometry around the metal approximates most closely to a trigonal dodecahedron for both the complexes. The three $\beta$-diketonate ligands coordinate to the $\mathrm{Eu}$ ion with Eu-O bond length ranging from 2.4187 to $2.4830 \AA$ and
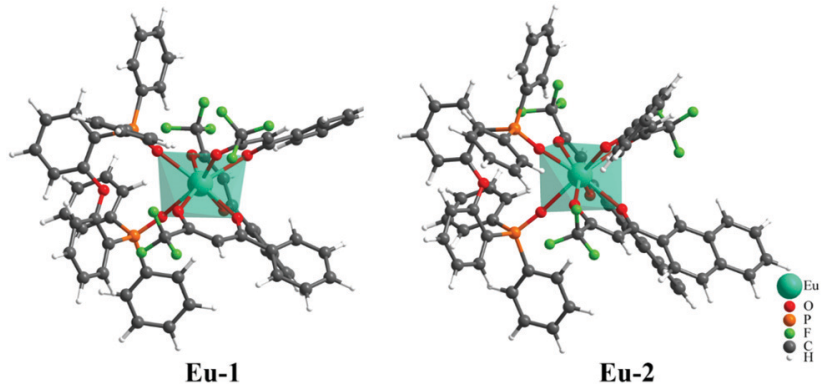

Fig. 1 Ground state geometry for complexes Eu-1 and Eu-2 calculated by the B3LYP/SVP/MWB52 level of theory. 
from 2.4152 to $2.5030 \AA$, for Eu-1 and Eu-2, respectively. The chelating DPEPO ligand coordinates to $\mathrm{Eu}$ ion with the $\mathrm{Eu}-\mathrm{O}$ bond lengths of 2.3937 and $2.4169 \AA$ and 2.3872 and $2.4321 \AA$, respectively, for the Eu-1 and Eu-2 complexes.

The Eu-O(DPEPO) bond distances found in the literature for related eight coordinate $\mathrm{Eu}(\mathrm{III})$ phosphine oxide complexes are in the range of 2.345-2.446 $\AA$ and are in agreement with those observed in the literature. ${ }^{28}$ The Eu-O bond distances are longer in Eu-2 compared to Eu-1. This could be attributed to the bulkier (napthayl vs. phenyl) nature of the $\beta$-diketone in the former, forcing the ligating $\mathrm{O}$ atoms to adopt longer distances to reduce the steric crowding around the metal center.

\subsection{Experimental and theoretical determination of photophysical properties}

The optical absorption spectra of the complexes were measured in DCM solution, as shown in Fig. 2a. The uncoordinated DPEPO displays two major absorption peaks at $228 \mathrm{~nm}(\varepsilon=$ $\left.48506 \mathrm{M}^{-1} \mathrm{~cm}^{-1}\right)$ and $290 \mathrm{~nm}\left(\varepsilon=8863 \mathrm{M}^{-1} \mathrm{~cm}^{-1}\right){ }^{2 a}$ The absorption spectra of the complexes exhibit combined absorption of the primary $\beta$-diketone and ancillary DPEPO ligand in DCM solution and the band shapes are similar with $\lambda_{\max }=$ $326 \mathrm{~nm}\left(30674 \mathrm{~cm}^{-1}\right)$ and $336 \mathrm{~nm}\left(29761 \mathrm{~cm}^{-1}\right)$ for Eu-1 and Eu-2, respectively. The absorption band-edge of the $\pi-\pi^{*}$ transition of Eu-1 is at around $382 \mathrm{~nm}\left(26187 \mathrm{~cm}^{-1}\right)$ while this is at

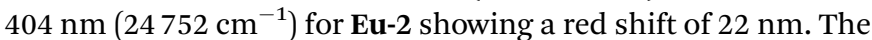
redshift could be attributed to the enhanced conjugation when a terminal phenyl is substituted with the naphthyl group in the case of Eu-2, thus resulting in less photobleaching. Fig. $2 b$ and c show the absorption spectra calculated using both the TD-DFT, CAM-B3LYP/SVP/MWB52 approach and the INDO/S-CIS semiempirical model from the B3LYP/SVP/MWB52 geometries. While the TD-DFT spectra effectively reproduced the redshift (Fig. 2b), this was not the case in the spectra obtained with the INDO/S-CIS model (Fig. 2c). By considering the effect of the DCM solvent implicitly in TD-DFT calculations, this effect does not cause a shift of the more intense absorption.

Table S2, ESI, $\uparrow$ shows the highest occupied molecular orbital (HOMO) and lowest unoccupied molecular orbital (LUMO) energy levels and $\Delta E_{\text {(номо-Lumo) }}$, respectively for the Eu-1 and Eu-2 calculated by different levels of theory. While the RM1 energies were calculated for the optimized geometry at the same computational level, the CAM-B3LYP energies were calculated by considering the optimized geometry at the B3LYP/ SVP/MWB52 level of theory. At the same level of theory, the Eu-2 complex has a higher $\Delta E_{\text {(номо-Luмо) }}$ than the one calculated for Eu-1. As suggested by Nüesch et al. ${ }^{29}$ the hole and electron trapping ability of dopants depend on the HOMO and LUMO energy levels of dopant and host molecules in the EL devices. Thus, these results indicate that both the complexes could be good candidates for trapping the charge in the EL devices. In the case of Eu-2, for instance, the singlet excited state with the higher oscillator strength relies on the contribution of 18 electronic transitions, making it difficult to determine the qualitative nature of the excited state. In order to elucidate the nature of the orbitals involved in the UV electronic absorption transitions regarding the singlet excited state of interest (higher oscillator strength) for Eu-1 and Eu-2, calculations of the natural transition orbitals (NTOs) were employed (Fig. 3). The NTO analysis offers a simple representation of the transition density between the ground and the excited state. It is possible to observe from Fig. 3 that the most important electronic transitions are $\pi \rightarrow \pi^{*}$ centered on the different $\beta$-diketone ligands. These transitions involve MOs energetically close to the HOMO and LUMO frontier MOs for both complexes. It is important to emphasize that the MO center on the DPEPO does not contribute to the most intense absorption band (Fig. 3). However, the DPEPO plays an important role in completing the coordination sphere of the $\operatorname{Ln}(\mathrm{III})$ ion and thus preventing the coordination of solvent molecule(s), which may enable the luminescence suppression via non-radiative decay. Simultaneously, imposing a highly asymmetric coordination geometry such as trigonal dodecahedral in our case is an important factor for enhancing the PLQY with large radiative rate constants. ${ }^{30}$

The PL properties of both the Eu-1 and Eu-2 were determined in DCM solution at RT (Fig. 4). In order to evaluate the detailed photophysical properties of the complexes and to maximize the steady-state emission, we first obtained the excitation spectra (Fig. S8, ESI $\dagger$ ) for both the complexes by monitoring
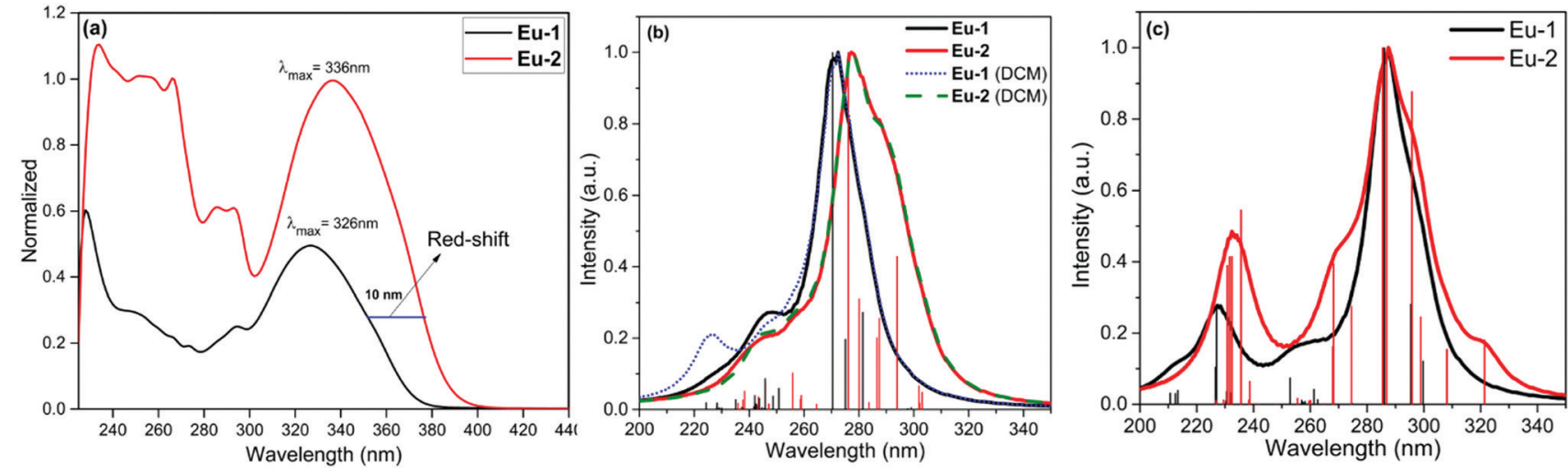

Fig. 2 (a) Electronic spectra of Eu-1 and Eu-2 in DCM at RT. Estimated electronic spectra by using different levels of theory for the complexes under investigation (b) CAM-B3LYP/SVP/MWB52//B3LYP/SVP/MWB52 with and without the effect of the DCM solvent, and (c) INDO/S-CIS//B3LYP/SVP/ MWB52. 

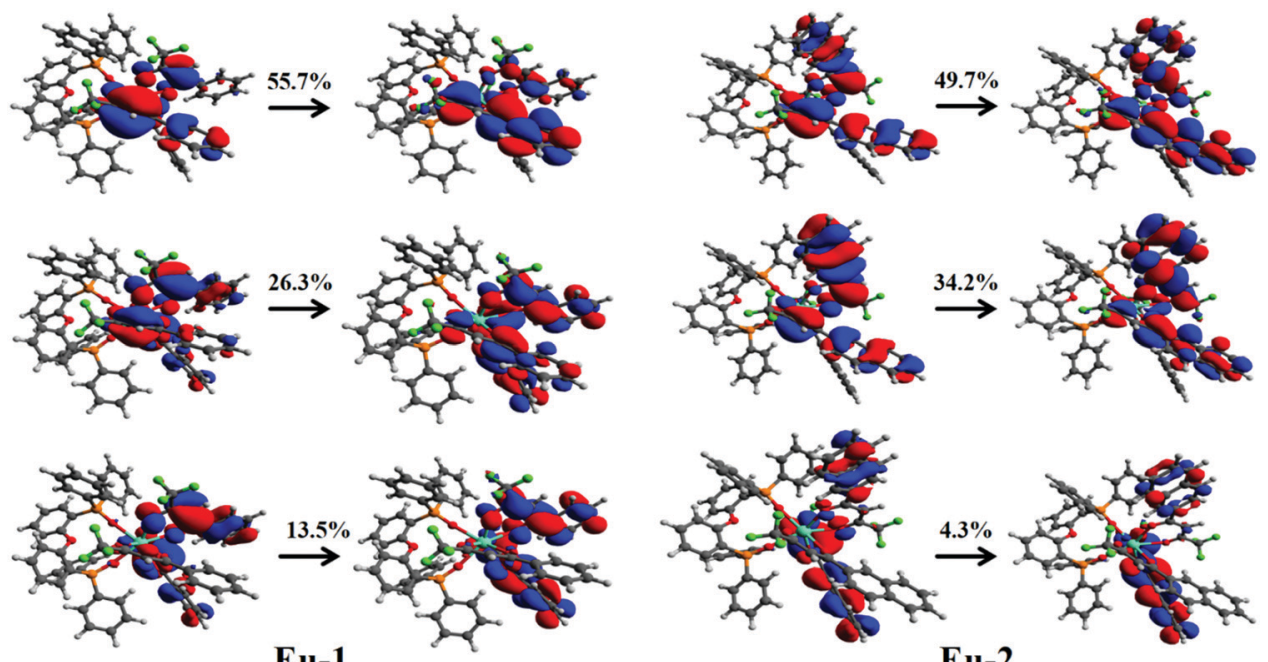

Eu-2

Fig. 3 Pictures of the NTOs mainly contributing to the singlet excited state with higher oscillator strength for Eu-1 (around $270.4 \mathrm{~nm}$ ) and Eu-2 (around $276.1 \mathrm{~nm}$ ). For each transition, the contribution percentages of the main NTOs are also included.

the ${ }^{5} \mathrm{D}_{0} \rightarrow{ }^{7} \mathrm{~F}_{2}$ emission transition. The excitation spectra of both complexes in DCM solution are similar and displayed a broadband in the region between $350 \mathrm{~nm}$ and $440 \mathrm{~nm}$ with a very faint intra-configurational $\mathrm{f}-\mathrm{f}$ transition at ${ }^{7} \mathrm{~F}_{0} \rightarrow{ }^{5} \mathrm{D}_{2}$ $\left(21505 \mathrm{~cm}^{-1} ; 465 \mathrm{~nm}\right)$, suggesting that the antenna mechanism as noted in similar $\left[\mathrm{Eu}(\mathrm{tfac})_{3} \mathrm{DPEPO}\right]$ and other related ternary $\beta$-diketonate complexes is functioning here. ${ }^{2 a, 5 c, 17}$ Moreover, the excitation spectra of Eu-2 exhibited a bathochromic shift, as noted in the electronic spectra of the complexes at RT and could be caused by the same effect.

The PL spectra shown in Fig. 4 for the complexes were obtained by exciting them at $\lambda_{\max }^{\mathrm{Ex}}$ mentioned in the figure caption. The spectra displayed essentially five well-resolved emission transitions emanating from the ${ }^{5} \mathrm{D}_{0}$ state to ${ }^{7} \mathrm{~F}_{J}(J=0,1,2,3$ and 4$)$. The data obtained for the parameters such as intensity, barycenter, and $\%$ contribution of each transition is gathered in Table S3, ESI. $\dagger$ A well-resolved and faint (\% contribution of $0.23-0.24 \%$ ) emission transition ${ }^{5} \mathrm{D}_{0} \rightarrow{ }^{7} \mathrm{~F}_{0}$ with FWHM of $1.17 \mathrm{~nm}$ and $1.55 \mathrm{~nm}$ (Table 1) for Eu-1 and Eu-2, respectively, can be seen in Fig. 4,

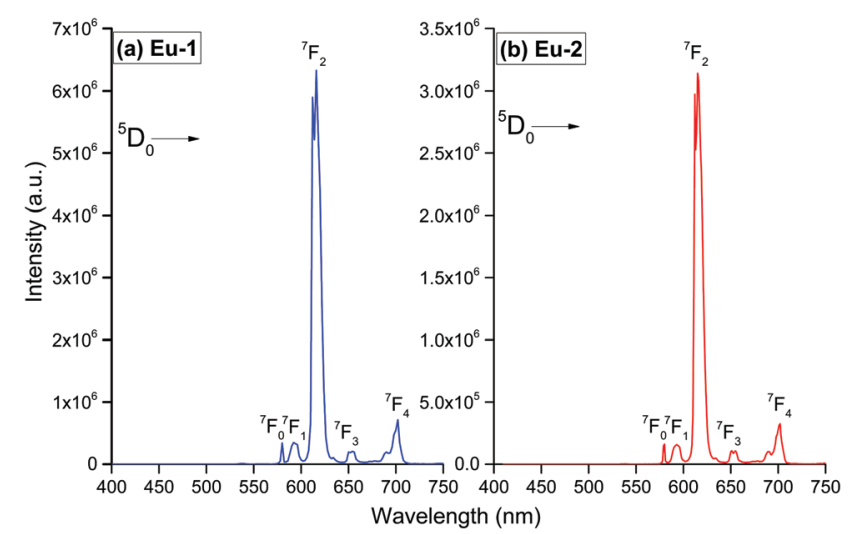

Fig. 4 Emission spectra of the Eu(III) complexes in DCM solution at RT (a) $\lambda_{\mathrm{Ex}}^{\max }=380 \mathrm{~nm}$ and (b) $\lambda_{\mathrm{Ex}}^{\max }=393 \mathrm{~nm}$. which suggests a single chemical environment around the $\mathrm{Eu}(\mathrm{III})$ ion $^{1 a, 2 a, 5 c, 17}$ in both complexes. The fourth emission transition ${ }^{5} \mathrm{D}_{0} \rightarrow{ }^{7} \mathrm{~F}_{3}$ follows the same mechanism as the ${ }^{5} \mathrm{D}_{0} \rightarrow{ }^{7} \mathrm{~F}_{0}$ transition, i.e., is not allowed by either mechanism, the magnetic-dipole (MD) and electric-dipole (ED) and contribute only $2.21-2.49 \%$ to the total integral emission output. The second MD transition ${ }^{5} \mathrm{D}_{0} \rightarrow{ }^{7} \mathrm{~F}_{1}$ and its intensity is independent of the $\mathrm{Eu}(\mathrm{III})$ coordination sphere; however, it varies with the change in the refractive index of the medium. ${ }^{5 c, 31}$ Thus it is used as a standard for the dipole strength and allows (will be discussed later) the determination of the natural radiative lifetime ( $\left.\tau_{\text {rad }}\right)$ for a given Eu-CC. The third transition ${ }^{5} \mathrm{D}_{0} \rightarrow{ }^{7} \mathrm{~F}_{2}$ in the series, ED is "hypersensitive" in nature, and so its intensity will change with the subtle change in the environment around the Eu(III) ion. The PL spectra are dominated by this narrow emission transition with FWHM of $(10.31 \mathrm{~nm})_{\mathbf{E u}-1}$ and $(9.66 \mathrm{~nm})_{\mathbf{E u}-2}$ (Table 1) of the total integral of the intensity $(82.77 \%)_{\mathbf{E u}-1}$ and $(83.44 \%)_{\mathbf{E u}-2}$ (Table S3, ESI $\dagger$ ) suggesting highly monochromatic bright red emission with CIE color coordinates $(0.672 ; 0.326)_{\mathbf{E u}-1}$ and $(0.673 ; 0.326)_{\mathbf{E u}-2}$ (Table 1, Fig. S9 and S10, ESI $\dagger$ ). The calculated CIE color coordinates obtained from the PL spectra are very similar to the National Television System Committee (NTSC: $x-0.67$; $y$ - 0.33), suggesting that these Eu-CCs could be a potential candidates as red components for the fabrication of EL devices. The higher total integral intensity of ED ${ }^{5} \mathrm{D}_{0} \rightarrow{ }^{7} \mathrm{~F}_{2}$ (\% contribution of $82-84 \%)$ transition compared to $\mathrm{MD}{ }^{5} \mathrm{D}_{0} \rightarrow{ }^{7} \mathrm{~F}_{1}$ transition indicates that forced $\mathrm{ED}$ and the dynamic coupling (DC) mechanism are dominant over MD. Furthermore, the PL spectra of both the complexes exhibit ${ }^{5} \mathrm{D}_{1} \rightarrow{ }^{7} \mathrm{~F}_{1}$ transition (Fig. S11 and S12, ESI $\dagger$ ). The PL spectrum of $\mathrm{Eu}(\mathrm{III})$ ion is quite informative because of the presence of $\mathrm{MD}^{5} \mathrm{D}_{0} \rightarrow{ }^{7} \mathrm{~F}_{1}$ transition and a simple analysis and calculations can give plenty of information together with experimentally observed lifetime $\left(\tau_{\mathrm{obs}}\right)$. For example, the intensity ratio $\left(R_{21}\right)$ is a measure of asymmetric environment around $\mathrm{Eu}(\mathrm{III})$ ion and a value higher than 0.7 could be attributed to the non-centrosymmetric and low 
Table 1 Experimental and theoretical photophysical parameters of Eu-1 and Eu-2 at RT in DCM

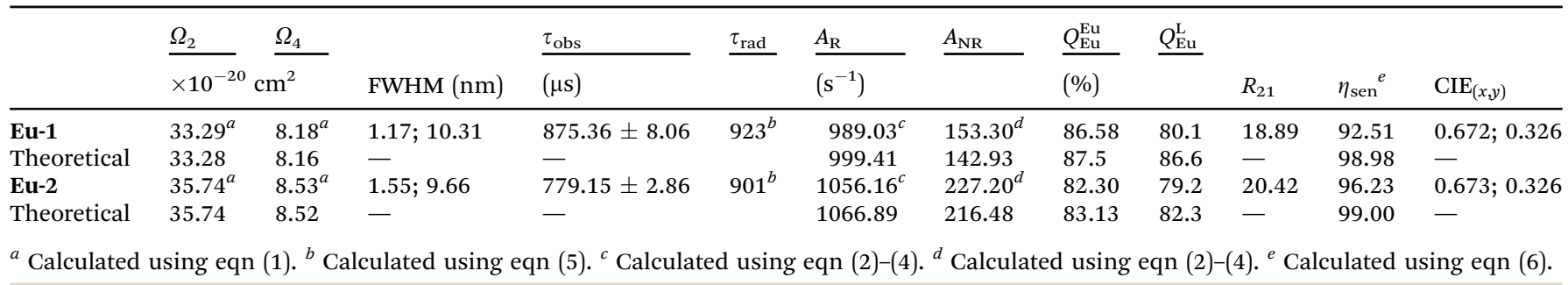

symmetric environment. The high value of $R_{21}=(18.89)_{\mathbf{E u - 1}}$ and $(20.42)_{\mathbf{E u - 2}}$, respectively (Table 1) suggests that the complexes are non-centrosymmetric with $C_{1}$ point group as determined by the ground state geometry of the complexes.

\subsection{J-O parameters, excited-state lifetime, and rate constants}

In order to get more information regarding the photophysical properties, the PL lifetime ( $\left.\tau_{\text {obs }}\right)$ of the ${ }^{5} \mathrm{D}_{0}$ excited state was determined in solution by exciting it at $\lambda_{\mathrm{Ex}}^{\max }$ and fitting the decay curves (Fig. S13 and S14, ESI $\dagger$ ) for Eu-1 and Eu-2 (Table 1). The fitting of the decay curves is in good agreement with monoexponential behavior $\left(\chi^{2}=1.00-1.01\right)$ suggesting the single dominant emitting species and attests the results of steady-state measurement where only one line is observed for the ${ }^{5} \mathrm{D}_{0} \rightarrow{ }^{7} \mathrm{~F}_{0}$ emission transition. The $\tau_{\mathrm{obs}}$ value of the present complexes display $\tau_{\text {obs }}=875.36 \pm 8.06$ and $779.15 \pm 2.86 \mu$ s for Eu-1 and Eu-2 (Table 1), respectively, typical for ternary europium complexes of $\beta$-diketone. As mentioned above, we further utilized the steady-state emission spectra and $\tau_{\mathrm{obs}}$ to get the information of radiative $\left(A_{\mathrm{R}}\right)$ and non-radiative $\left(A_{\mathrm{NR}}\right)$ decay constants with the help of eqn (2)-(5). The data obtained are gathered in Table 1 . It is very clear that the Eu-2 complex displayed a marginally larger value of the $A_{\mathrm{NR}} \approx 227.20 \mathrm{~s}^{-1}$ compared to Eu-1, $A_{\mathrm{NR}} \approx 153.30 \mathrm{~s}^{-1}$ which could be due to the presence of the higher number of $\mathrm{C}-\mathrm{H}$ oscillators in the case of Eu-2. The PLQY of the complexes in solution exhibited very large values of 80.1 and $79.2 \%$ and sensitization $\left(\eta_{\text {sen }}\right)$ efficiency of 92.51\% and 96.23\% for Eu-1 and Eu-2, respectively (Table 1). Finally, $\Omega_{2}$ and $\Omega_{4}$ were calculated (Table 1), the high values of $\Omega_{2}=33.29-35.74 \times 10^{-20} \mathrm{~cm}^{2}$ suggest that the Eu(III) ion in both the complexes is surrounded by a highly polarizable environment. ${ }^{1 a}$ The parameter $\Omega_{4}$ is related to the rigidity and long-range effects such as hydrogen bonding, $\pi-\pi$ stacking and substantially large value $\left(\Omega_{4} \approx 8.18 \times 10^{-20} \mathrm{~cm}^{2}\right)_{\text {Eu-1 }}$ and $\left(\Omega_{4} \approx 8.53 \times 10^{-20} \mathrm{~cm}^{2}\right)_{\text {Eu-2 }}$ is an indication of the presence of these effects. ${ }^{5 c}$ Zero Differential Overlap (ZDO) electronic densities $(q)$ and electrophilic superdelocalizabilities (SE) calculated with RM1, by considering the B3LYP/SVP/MWB52 geometry of each atom directly coordinated to the Eu(III) ion, in Eu-1 and Eu-2, together with the corresponding charge factors $g$ and polarizabilities $\alpha$ from the fitting (Table S4, $\mathrm{ESI} \dagger$ ). Moreover, through a series of tests using crystallographicallyrelated structures, it has been shown that the ratio between adjustable $D$ and $C$ parameters has a value greater than unity where the experimental intensity parameters were appropriately adjusted. ${ }^{32}$ Furthermore, the forced ED contribution to the intensity parameters $\left(\Omega_{\lambda}^{\mathrm{FED}}\right)$ was calculated using the $Q, D$, and $C$ values, and $\Omega_{\lambda}^{\mathrm{FED}}$ are important for the calculation of the ET rates related to the MM mechanism (eqn (9)).

\subsection{Intermolecular energy transfer (IET) process}

For an efficient sensitization of Ln(III) ion emissions in organolanthanide complexes, an appropriate energy difference between the triplet state of organic ligands and the emitting levels of $\mathrm{Ln}$ (III) ions must exist, which is $>2500 \mathrm{~cm}^{-1}$ for $\mathrm{Eu}(\mathrm{III})$ ion. ${ }^{1,2 a, 33}$ This criterion is satisfied by both complexes. The energy difference corresponds to the $\Delta$ parameter in the eqn (11), and this equation contains the dependence of the ET rate on the triplet and singlet energy of the coordinated ligands. Table 2 shows the lowest triplet energy for both complexes as well as the electron transition configurations mainly contributing to the state. Although structurally different, the Eu-1 and Eu-2 have only a marginal difference in energy for the lowest triplet state.

Fig. 5 shows the greater involvement of the MOs localized on the $\beta$-diketonate ligands and thus clearly indicates that the $\beta$-diketonate ligands are of great importance for the lowest triplet state in both complexes. Additionally, it also indicates that the PO-ligand does not play a crucial role in the luminescence sensitization. This observation is further supported by the TD-DFT work of Congiu et al., ${ }^{34}$ on the [Eu(hfaa) ${ }_{3}$ DPEPO] complex (hfaa $=$ hexafluoroacetylacetone) where they reported similar behavior i.e., the excited singlet-triplet is located on the $\beta$-diketonate ligand.

The ET rates were calculated using the values obtained employing the LUMPAC software package ${ }^{26}$ and are summarized in Table 3. Eu-1 was chosen as a representative for the discussion. The main accepting levels for the $\mathrm{Eu}(\mathrm{III})$ ion ${ }^{5} \mathrm{D}_{4}\left(\sim 27586 \mathrm{~cm}^{-1}\right)$, ${ }^{5} \mathrm{D}_{1}\left(\sim 19027 \mathrm{~cm}^{-1}\right)$, and ${ }^{5} \mathrm{D}_{0}\left(\sim 17293 \mathrm{~cm}^{-1}\right)$ are well above the

Table 2 Electron transition configurations for the lowest triplet state $\left(T_{1}\right)$ of Eu-1 and Eu-2

\begin{tabular}{|c|c|c|c|}
\hline & Major contribution & & Major contribution \\
\hline
\end{tabular}



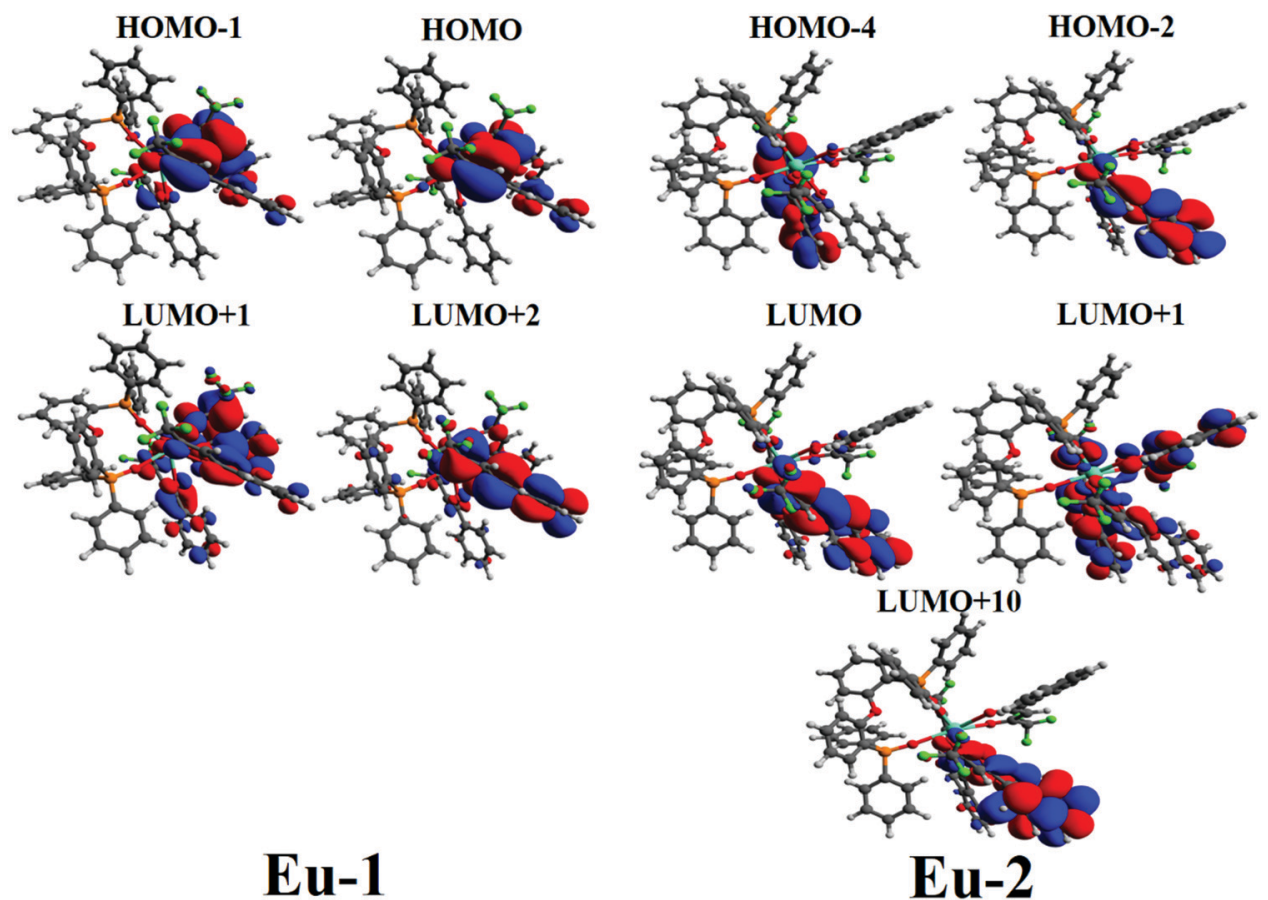

Fig. 5 CAM-B3LYP/SVP/MWB52 molecular orbitals mainly contributing to the lowest triplet state of Eu-1 and Eu-2.

Table 3 ET rates calculated by the Malta's model for the Eu-1 and Eu-2. The ligand state energies considered were those calculated with the CAMB3LYP/SVP/MWB52//B3LYP/SVP/MWB52 method

\begin{tabular}{lllllll}
\hline Complex & Ligand state $\left(\mathrm{cm}^{-1}\right)$ & & $4 \mathrm{f}$ state $\left(\mathrm{cm}^{-1}\right)$ & ET rate $\left(W_{\mathrm{ET}}\right)\left(\mathrm{s}^{-1}\right)$ & Energy back-transfer $\left(W_{\mathrm{BT}}\right)$ rate $\left(\mathrm{s}^{-1}\right)$ & Quantum yield $(\%)$ \\
\hline Eu-1 & Singlet $(36977.2)$ & $\rightarrow$ & ${ }^{5} \mathrm{D}_{4}(27586)$ & $W_{\text {ET1 }}=9.1 \times 10^{3}$ & $W_{\text {BT1 }}=0$ \\
& Triplet $(22413.7)$ & $\rightarrow$ & ${ }^{5} \mathrm{D}_{1}(19027)$ & $W_{\text {ET2 }}=1.2 \times 10^{10}$ & $W_{\text {BT2 }}=1.0 \times 10^{3}$ \\
& Triplet $(22413.7)$ & $\rightarrow$ & ${ }^{5} \mathrm{D}_{0}(17293)$ & $W_{\text {ET3 }}=8.5 \times 10^{9}$ & $W_{\text {BT3 }}=1.8 \times 10^{-1}$ \\
& & & & & \\
Eu-2 & Singlet $(36220.4)$ & $\rightarrow$ & ${ }^{5} \mathrm{D}_{4}(27586)$ & $W_{\text {ET1 }}=1.9 \times 10^{2}$ & $W_{\text {BT1 }}=0$ \\
& Triplet $(22280.4)$ & $\rightarrow$ & ${ }^{5} \mathrm{D}_{1}(19027)$ & $W_{\text {ET2 }}=1.9 \times 10^{8}$ & $W_{\text {BT2 }}=3.2 \times 10$ \\
& Triplet $(22280.4)$ & $\rightarrow$ & ${ }^{5} \mathrm{D}_{0}(17293)$ & $W_{\text {ET3 }}=1.4 \times 10^{8}$ & $W_{\text {BT3 }}=5.8 \times 10^{-3}$
\end{tabular}

singlet and the triplet state (Table 3). The selection rules state that ET from singlet to ${ }^{5} \mathrm{D}_{4}\left({ }^{5} \mathrm{D}_{4} \leftarrow{ }^{7} \mathrm{~F}_{0}\right)$ excitation in $\mathrm{Eu}(\mathrm{III})$ ion occurs through a multipolar mechanism (MM) and the lower value suggests that MM mechanism is not the dominant ET process (Table 3). The ET involving the channels, triplet $\rightarrow{ }^{5} \mathrm{D}_{1}$ and triplet $\rightarrow{ }^{5} \mathrm{D}_{0}$, are related to the ${ }^{5} \mathrm{D}_{1} \leftarrow{ }^{7} \mathrm{~F}_{0}$ and ${ }^{5} \mathrm{D}_{0} \leftarrow{ }^{7} \mathrm{~F}_{1}$ excitations respectively, considering that the thermally populated ${ }^{7} \mathrm{~F}_{1}$ state proceeds through an ExM (Dexter mechanism). ${ }^{2 b}$ A representative Jablonski energy diagram representing the most probable ET channels is displayed in Fig. 6. The higher values of $W_{\mathrm{ET}}$ rate of $\mathrm{T}_{1} \rightarrow{ }^{5} \mathrm{D}_{1} \approx 1.2 \times 10^{10} \mathrm{~s}^{-1}$ and $\mathrm{T}_{1} \rightarrow{ }^{5} \mathrm{D}_{0} \approx 8.5 \times$ $10^{9} \mathrm{~s}^{-1}$ suggest that the direct ligand-to-metal ET is dominated by the ExM. Interestingly, Eu-2 showed marginally smaller value of $W_{\mathrm{ET}}$ rate of $\mathrm{T}_{1} \rightarrow{ }^{5} \mathrm{D}_{1} \approx 1.9 \times 10^{8} \mathrm{~s}^{-1}$ and $\mathrm{T}_{1} \rightarrow{ }^{5} \mathrm{D}_{0} \approx 1.4 \times$ $10^{8} \mathrm{~s}^{-1}$ compared to Eu-1. A plausible explanation for this observation could be attributed to the longer $R_{\mathrm{L}}$ value ( $c a .5 \AA$ ) for Eu-2, which is due to the extended conjugation of the substituent (naphthyl) on Eu-2. Thus, the electronic density involved in the ligand-metal ET is further away from the energy acceptor center in Eu-2 compared to Eu-1 (Fig. 5). Furthermore, since the enthalpy of formation difference $\left(\Delta H_{\mathrm{F}}\right)$ among some of the different geometries calculated by RM1 model are very similar, it is worthwhile to evaluating the effect of the geometry on the excited state energies and their corresponding $R_{\mathrm{L}}$ distances. We applied the INDO/S-CIS model and the obtain data are summarized in Table S5, ESI, $\dagger$ it shows small variation for the different geometries and therefore the main results regarding the ET process remain unaltered in the complexes. Moreover, the solvent effect provides a very modest difference in the most relevant quantities for the ET process $\left(R_{\mathrm{L}, \sin g}, E_{\text {sing }}, R_{\mathrm{L}, \text { trip }}\right.$, and $\left.E_{\text {trip }}\right)$ in comparison with the same quantities without solvent (Table S6, ESI $\dagger$ ). Because the ligand triplet state is well above the ${ }^{5} \mathrm{D}_{0}$ and ${ }^{5} \mathrm{D}_{1}$ levels of the Eu(III) ion, the direct ET is favored and a small energy difference is not enough for significantly changing the ET.

The theoretical PLQY for Eu-1 and Eu-2 are shown in Table 3. Theoretical PLQY depends on the ET rates, $A_{\mathrm{R}}$ and $A_{\mathrm{NR}}$ decay rates, in addition to the other non-radiative rates, whose typical experimental values are presented in Fig. 6. The normalized populations of the ligand and metal ion states 


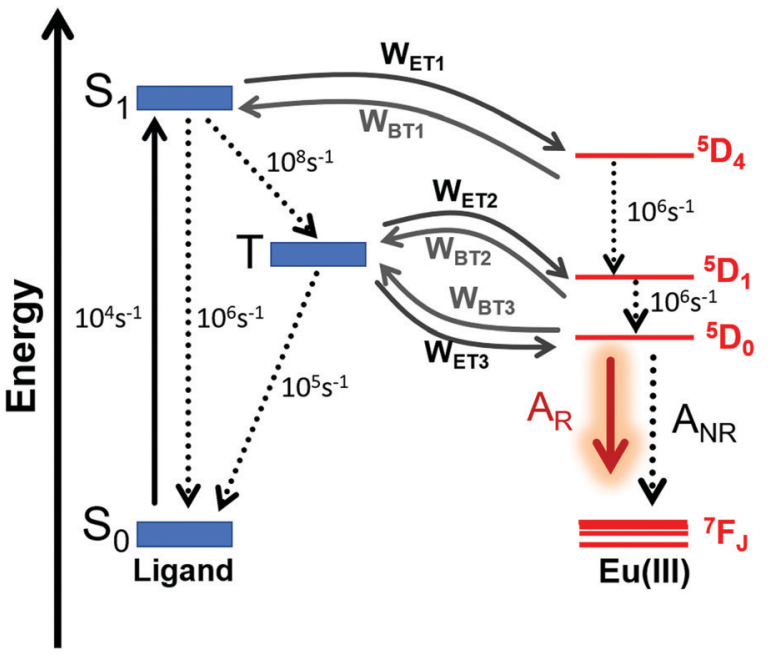

Fig. 6 Schematic energy-level diagram, ET processes, and transfer rates considered for both complexes. The rates shown are typical experimental values for the non-radiative rates in coordination compounds ${ }^{35}$ and are implemented into LUMPAC. ${ }^{26}$ Please see Table 1 for $A_{R}$ and $A_{N R}$, and Table 3 for $W_{E T}$ and $W_{B T}$ values for both complexes.

considered in the PL process are calculated by using all rates and, in particular, the most important populations are those associated with states $\mathrm{S}_{0}$ and ${ }^{5} \mathrm{D}_{0}$ (eqn (S10), ESI $\dagger$ ). One reason of the direct transfer rate for the two complexes to be greatly favored, such as indicated by the theoretical ET modeling, the sensitization efficiency for each complex is large considering either experimental or theoretical value (Table 1). In this way, the most relevant ET channels were considered in the theoretical ET modeling.

\subsection{EL performance of Eu-1 and Eu-2}

The impressive photophysical properties of the two europium complexes such as narrow ED ${ }^{5} \mathrm{D}_{0} \rightarrow{ }^{7} \mathrm{~F}_{2}$ (FWHM $=9.66-$ $10.31 \mathrm{~nm})$ emission transition with the total integral contribution (82.00-83.44\%) and color coordinates (CIE; 0.673; 0.326) suggest that the complexes could be utilized as efficient emitting materials in EL devices. To characterize the EL properties, Eu-1 and Eu-2 were used as emitters to fabricate single- and double EML devices with the following general device configuration (please see the $\mathrm{ESI} \dagger$ for further details).

Single-EML device. ITO/HAT-CN (6 nm)/HAT-CN (0.2 wt\%): TAPC $(50 \mathrm{~nm}) / \mathbf{E u}-1$ or Eu-2 $(x$ wt\%): 26DCzPPy $(10 \mathrm{~nm}) /$ Tm3PyP26PyB (60 nm)/LiF (1 nm)/Al (100 nm).

Double-EML device. ITO/HAT-CN (6 nm)/HAT-CN (0.2 wt\%): TAPC (50 nm)/Eu-1 or Eu-2 $(x$ wt\%): TcTa $(10 \mathrm{~nm}) / \mathbf{E u}-\mathbf{1}$ or Eu-2 ( $x$ wt $\%):$ 26DCzPPy $(10 \mathrm{~nm}) / \mathrm{Tm} 3 \mathrm{PyP} 26 \mathrm{PyB}(60 \mathrm{~nm}) / \mathrm{LiF}(1 \mathrm{~nm}) /$ $\mathrm{Al}(100 \mathrm{~nm})$.

EL devices based on Eu-1 and Eu-2 were fabricated by thermal evaporation under vacuum, which was facilitated by high thermal stability of the complexes $\left(318-320{ }^{\circ} \mathrm{C}\right) .{ }^{12}$ The doping concentration for europium complexes was modulated to be $3.0 \mathrm{wt} \%$, $4.0 \mathrm{wt} \%$ and $5.0 \mathrm{wt} \%$, respectively, As the doping concentration
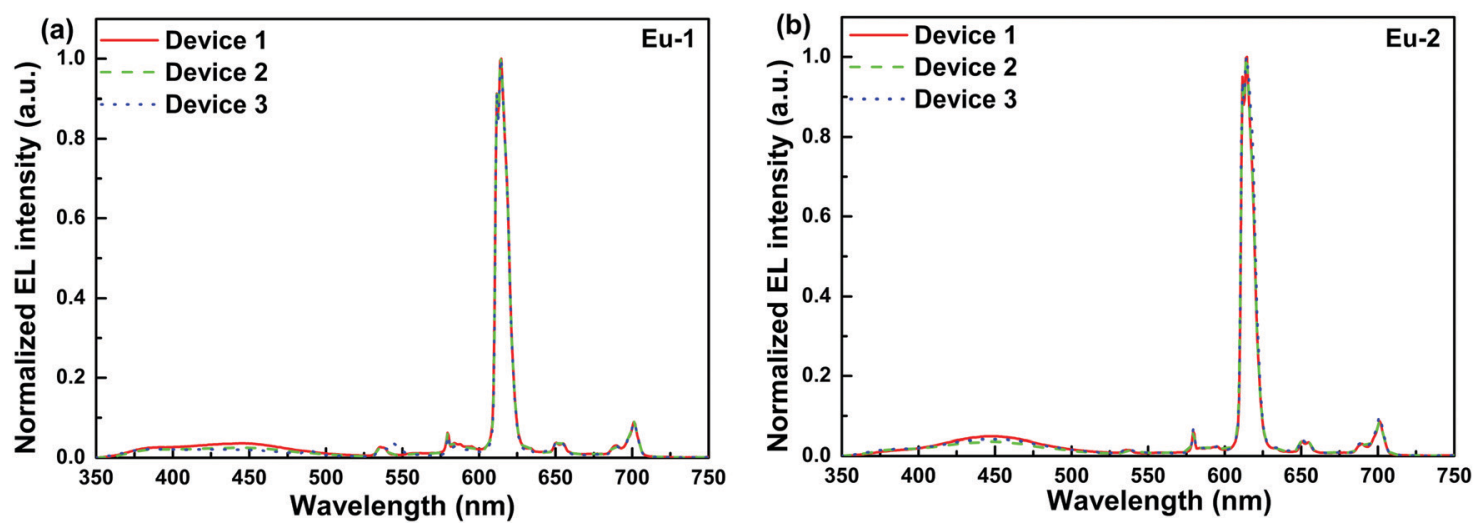

Fig. 7 Normalized EL spectra of the single-EML devices 1, 2 and 3 of (a) Eu-1 and (b) Eu-2 at $10 \mathrm{~mA} \mathrm{~cm}^{-2}$.

Table 4 Key properties of single EML EL device 1, 2 and $\mathbf{3}$ for Eu-1 and Eu-2

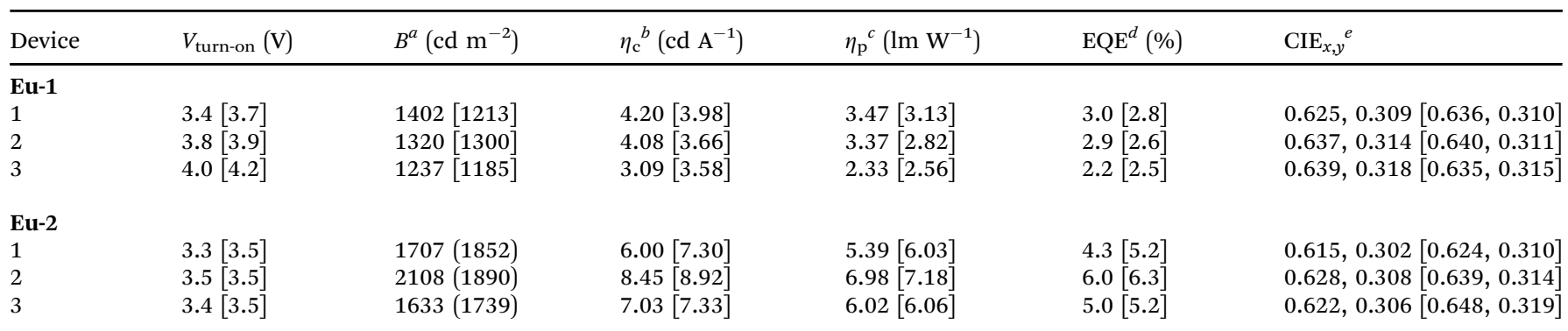

The values in square brackets are EL performances of the double layer devices $\mathbf{4}, \mathbf{5}$ and $\mathbf{6}$ of Eu-1 and Eu-2 complexes. ${ }^{a}$ The data for maximum brightness $(B)$. ${ }^{b}$ Maximum current efficiency $\left(\eta_{\mathrm{c}}\right) .{ }^{c}$ Maximum power efficiency $\left(\eta_{\mathrm{p}}\right) \cdot{ }^{d}$ Maximum external quantum efficiency $(\mathrm{EQE}) .{ }^{e} \mathrm{CIE}_{x y}$ at $10 \mathrm{~mA} \mathrm{~cm}{ }^{-2}$. 
increases, the evaporation temperature increases gradually from 140 to $151{ }^{\circ} \mathrm{C}$ and 147 to $159{ }^{\circ} \mathrm{C}$ for Eu-1 and Eu-2, respectively. The low thermal evaporation temperature of the complexes thus ensures negligible decomposition of the complexes during the thermal evaporation process. The normalized EL spectra of Eu-1 and Eu-2 based single and double EML devices are shown in Fig. 7 and Fig. S15 and S16, ESI. $\dagger$

The spectra displayed extremely sharp EL similar to the PL spectra for both the complexes and is dominated by ED transition at $615 \mathrm{~nm}$ characteristic for the $\mathrm{Eu}(\mathrm{III})$ ion as observed in the
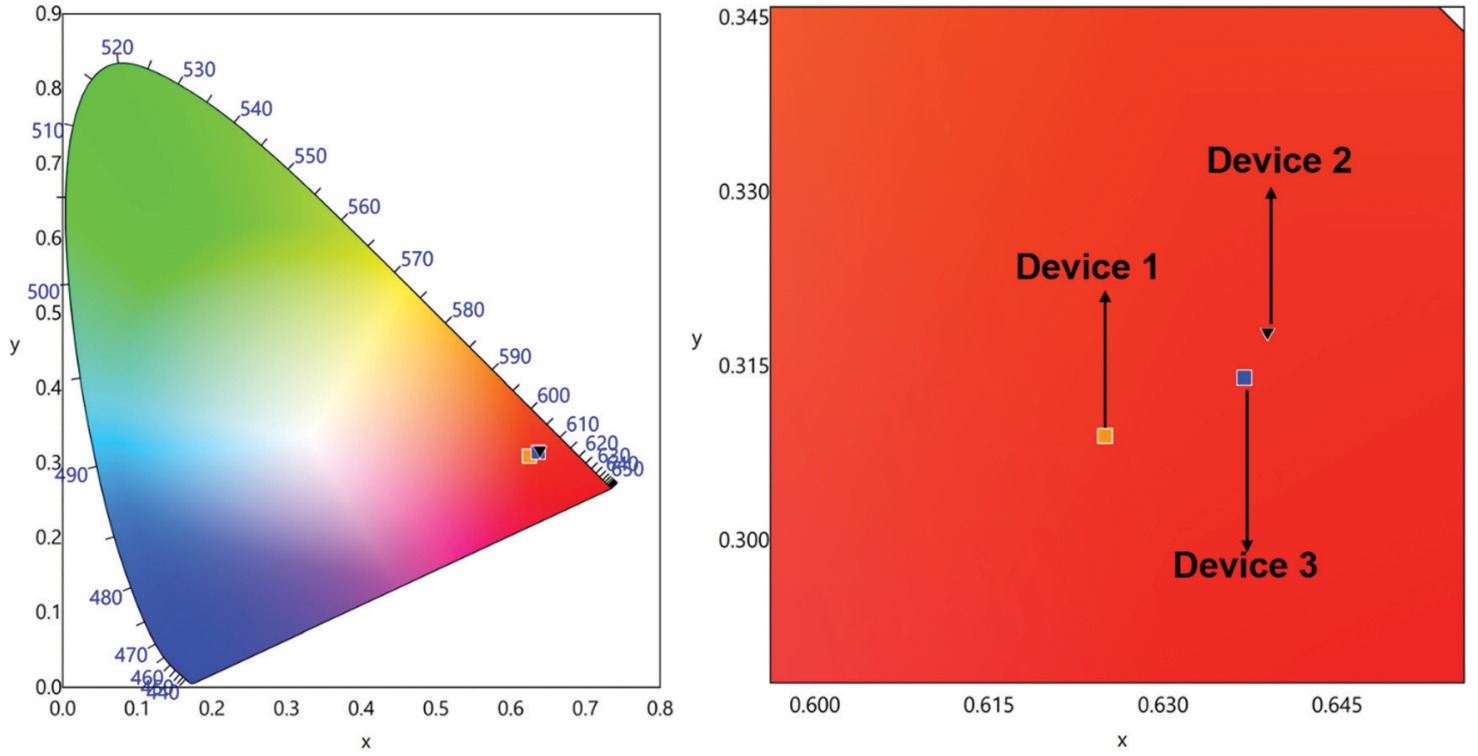

Fig. $8 \mathrm{CIE} 1931$ chromaticity diagrams of single EML devices 1,2 and $\mathbf{3}$ of Eu-1 with a magnified view at $10 \mathrm{~mA} \mathrm{~cm}$.
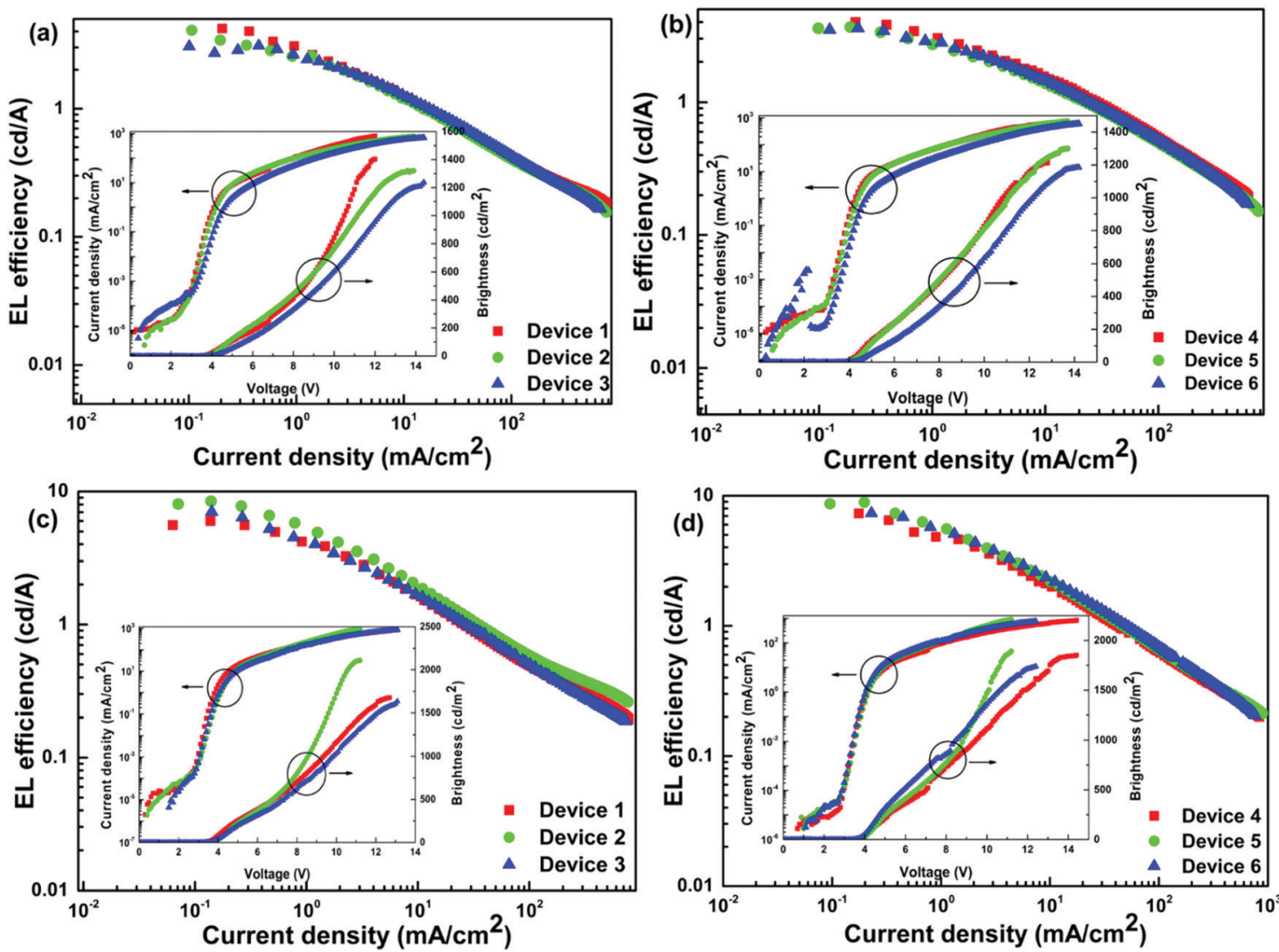

Fig. 9 EL efficiency-current density characteristics of devices. Inset: Current density-brightness-voltage characteristics of their respective devices. ( $a$ and b) Single and double EML devices of Eu-1 while (c and d) are single and double EML devices of Eu-2 operating at $10 \mathrm{~mA} \mathrm{~cm}^{-2}$. 
steady-state emission analysis. Moreover, they displayed minor high-lying emissions in the range between 400 and $500 \mathrm{~nm}$ of host emission, which decreases as the doping concentration increases. The devices emitted pure red emission in spite of the presence of the very faint host emission with $(\mathrm{CIE})_{x, y}$ color coordinates of $(x=0.622, y=0.306)-(x=0.648, y=0.319)$ (Table 4, Fig. 8 and Fig. S17-S19, ESI $\dagger$ ). In the device luminescence process for the present complexes, we speculate that both carriers' trapping and Förster ET may co-exist simultaneously ${ }^{36}$ since the EL spectra (Fig. 7) in the range of $500-750 \mathrm{~nm}$ resembles well the PL spectra (Fig. 4) except the presence of a faint broadband in the range between 350 and $450 \mathrm{~nm}$ due to the incomplete ET from the host material.

The EL efficiency and current density curves together with the voltage $(V)$-brightness $(B)$ and current density curves as an inset are shown in Fig. 9. The detailed EL performance parameters such as $B, \eta_{\mathrm{c}}, \eta_{\mathrm{p}}$ and EQE of single- and double-EML devices are gathered in Table 4. For Eu-1, at the optimum doping concentration of $4.0 \mathrm{wt} \%$, the single EML displayed, $B=1320 \mathrm{~cd} \mathrm{~m}^{-2}, \eta_{\mathrm{c}}=4.08 \mathrm{~cd} \mathrm{~A}^{-1}, \eta_{\mathrm{p}}=3.37 \mathrm{~lm} \mathrm{~W}^{-1}$ with an impressive $\mathrm{EQE}=2.9 \%$ at very low $V_{\text {turn-on }}=3.8 \mathrm{~V}$ with $(\mathrm{CIE})_{x, y}=$ $0.637,0.314$ in the pure red region. The double-EML devices at the same doping concentration do not exhibit any significant change except for a marginal change in the brightness. For Eu-2 at the similar optimum concentration, the EL performance of the single EML device showed outstanding results with $B=2108 \mathrm{~cd} \mathrm{~m}^{-2}, \eta_{\mathrm{c}}=8.45 \mathrm{~cd} \mathrm{~A}^{-1}, \eta_{\mathrm{p}}=6.98 \mathrm{~lm} \mathrm{~W}^{-1}$ with an impressive $\mathrm{EQE}=6.0 \%$ at very low $V_{\text {turn-on }}=3.5 \mathrm{~V}$ with $(\mathrm{CIE})_{x, y}=$ $0.628,0.308$. As suggested by Xu et al. ${ }^{37}$ the $V_{\text {turn-on }}$ is relative to the carrier injection and transport ability of each layer. The low

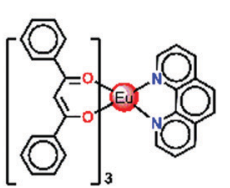

Eu-3

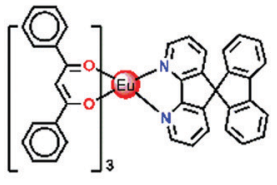

Eu-8

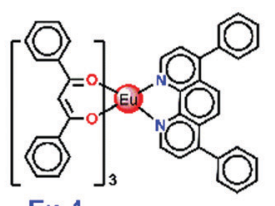

Eu-4

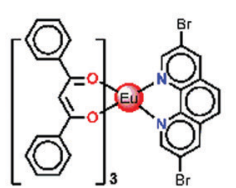

Eu-5

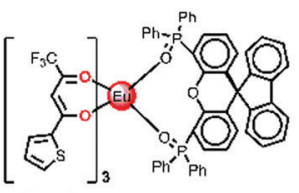

Eu-6

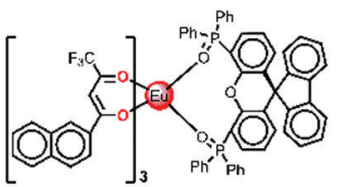

Eu-7

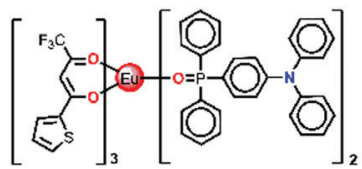

Eu-10

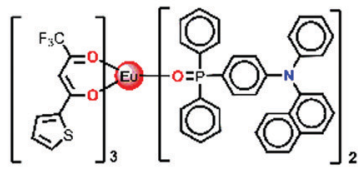

Eu-11
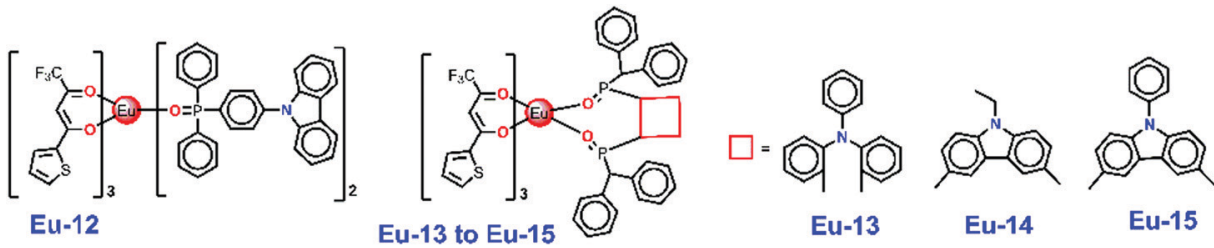

Eu-15

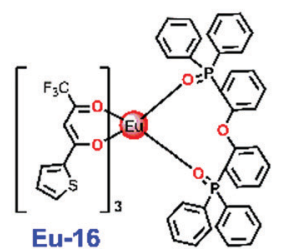

Chart 2 Chemical structure of the reported efficient ternary Eu-CCs.

Table 5 Comparative EL performances of reported Eu-CCs (their chemical structures are shown in Chart 2)

\begin{tabular}{|c|c|c|c|c|c|c|c|}
\hline Emitting layer & $V_{\text {turn-on }}(\mathrm{V})$ & $B^{a}\left(\mathrm{~cd} \mathrm{~m}^{-2}\right)$ & $\eta_{\mathrm{c}}^{b}\left(\mathrm{~cd} \mathrm{~A}^{-1}\right)$ & $\operatorname{EQE}^{d}(\%)$ & $\eta_{\mathrm{p}}^{c}\left(\operatorname{lm~} \mathrm{W}^{-1}\right)$ & $\mathrm{CIE}_{x, y}^{e}$ & Ref. \\
\hline Eu-2 $(3 \mathrm{wt} \%): 26 \mathrm{DCzPPy}(10 \mathrm{~nm})$ & 3.5 & 2108 & 8.45 & 6.0 & 6.98 & $0.628,0.308$ & This work \\
\hline Eu-3 (10 wt\%): TCTA:Bphen & 2.9 & - & 3.85 & 2.12 & 2.26 & - & 16 \\
\hline Eu-5 (90 nm): CBP: PBD & 12 & 278 & - & 1.45 & - & - & $38^{b}$ \\
\hline Eu-6 (90 nm): CBP: PBD & - & - & 3.2 & 2.40 & 0.6 & $0.65,0.32$ & $38^{c}$ \\
\hline Eu-7 (90 nm): CBP: PBD & - & - & 5.1 & 3.70 & 1.0 & $0.66,0.33$ & $38^{c}$ \\
\hline Eu-10 $(40 \mathrm{~nm})$ & 5.7 & - & 5.07 & 3.20 & 3.62 & - & 15 \\
\hline Eu-11 (40 nm) & 5.0 & - & 5.88 & 3.71 & 3.69 & - & 15 \\
\hline Eu-12 (40 nm) & 8.4 & - & 3.25 & 2.08 & 1.23 & - & 15 \\
\hline Eu-13: CBP (40 nm, 10\%) & 6.0 & 945 & - & 2.96 & - & - & 37 \\
\hline Eu-14: CBP (40 nm, 10\%) & 7.6 & 1276 & - & 3.54 & - & - & 37 \\
\hline Eu-15: CBP (40 nm, 10\%) & 7.2 & 1163 & N/A & 3.20 & - & - & 37 \\
\hline Eu-16: CBP (30 nm, 10\%) & 7 & 632 & 4.58 & 2.89 & 2.89 & - & 14 \\
\hline
\end{tabular}

Where, BPhen = 4,7-diphenyl-1,10-phenanthroline; PBD = 2-(tert-butylphenyl)-5-biphenylyl-1,3,4-oxadiazole; CBP = 4,4' $-N, N^{\prime}$-dicarbazole-biphenyl . ${ }^{a}$ The data for maximum brightness $(B) .{ }^{b}$ Maximum current efficiency $\left(\eta_{\mathrm{c}}\right) .{ }^{c}$ Maximum power efficiency $\left(\eta_{\mathrm{p}}\right) .{ }^{d}$ Maximum external quantum efficiency (EQE). ${ }^{e} \mathrm{CIE}_{x, y}$ at $10 \mathrm{~mA} \mathrm{~cm}{ }^{-2}$. 
$V_{\text {turn-on }}$ and high EL efficiency of Eu-2 could be caused by the barrier-free carrier injection, balanced carrier transport and recombination coupled with simultaneous high ET efficiency from the exciplex host to Eu-2. Interestingly the device based on Eu-2 outperformed many of the ternary europium complexes (Chart 2) reported ${ }^{14,15,37,38}$ in the literature as can be seen in a comparative Table 5. In fact, this is the best performance to the best of our knowledge.

\section{Conclusion}

In conclusion, we have synthesized two highly efficient red emitting complexes Eu-1 and Eu-2 with PLQY values as large as $80 \%$. The EL performance of the single EML device of Eu-2 exhibited bright red EL with the EQE of $6.0 \%$ and $B=2108 \mathrm{~cd} \mathrm{~m}^{-2}, \eta_{\mathrm{c}}=8.45 \mathrm{~cd} \mathrm{~A}^{-1}, \eta_{\mathrm{p}}=6.98 \mathrm{~lm} \mathrm{~W}^{-1}$ at very low $V_{\text {turn-on }}=3.5 \mathrm{~V}$ which are the best reported for devices with Eu-CC as emitters. Moreover, it is important to note that doping concentrations as low as $4 \mathrm{wt} \%$ generated the best EL performance compared to other red-emitting europium-based OLEDs reported in the literature (Chart 2). To the best of our knowledge, this is the best EL performance shown by a Eu-CC. Furthermore, the single EML device makes the fabrication of EL devices easy and decreases the manufacturing cost as well as the time taken to fabricate them, making these complexes important EL materials for real-life applications. Work on new phospho-oxide type ligand with large bite angle to impose asymmetric coordination geometry around Eu(III) and optimization of the device structures is in progress in our laboratory to further improve the device efficiency of the red emitting OLEDs.

\section{Conflicts of interest}

The authors declare no conflicts of interest.

\section{Acknowledgements}

MSK acknowledges His Majesty's Trust Fund for Strategic Research (Grant No. SR/SQU/SCI/CHEM/16/02) for funding. RI thanks HM's Trust Fund for a postdoctoral fellowship. WYW thanks the Hong Kong Polytechnic University (1-ZE1C) and the Endowed Professorship in Energy from Ms Clarea Au (847S) for the financial support. WFO thanks PIBIC/CNPq/UFS (02/2019 COPES/POSGRAP/UFS) for a scientific initiation fellowship. LZ is grateful to the financial aid from National Natural Science Foundation of China (21771172), Youth Innovation Promotion Association of Chinese Academy of Sciences (2013150). PRR is grateful to the Engineering and Physical Sciences Research Council (EPSRC) for continued funding (Grant EP/K004956/1).

\section{References}

1 (a) A. Haque, L. Xu, R. A. Al-Balushi, M. K. Al-Suti, R. Ilmi, Z. Guo, M. S. Khan, W. Y. Wong and P. R. Raithby, Chem. Soc. Rev., 2019, 48, 5547-5563; (b) R. Ilmi, E. Tseriotou,
P. Stylianou, Y. A. Christou, I. Ttofi, N. Dietis, C. Pitris, A. D. Odysseos and S. N. Georgiades, Mol. Pharmaceutics, 2019, 16, 4260-4273; (c) R. Ilmi, I. Juma Al-busaidi, A. Haque and M. S. Khan, J. Coord. Chem., 2018, 71, 3045-3076; (d) C. D. S. Brites, A. Millán and L. D. Carlos, in Handbook on the Physics and Chemistry of Rare Earths, ed. B. JeanClaude and P. K. Vitalij, Elsevier, 2016, vol. 49, pp. 339-427; (e) C.-L. Ho and W.-Y. Wong, Coord. Chem. Rev., 2013, 257, 1614-1649; $(f)$ H. Xu, R. Chen, Q. Sun, W. Lai, Q. Su, W. Huang and X. Liu, Chem. Soc. Rev., 2014, 43, 3259-3302. 2 (a) R. Ilmi, M. S. Khan, W. Sun, L. Zhou, W.-Y. Wong and P. R. Raithby, J. Mater. Chem. C, 2019, 7, 13966-13975; (b) R. Ilmi, M. S. Khan, Z. Li, L. Zhou, W.-Y. Wong, F. Marken and P. R. Raithby, Inorg. Chem., 2019, 58, 8316-8331; (c) B. Francis, M. M. Nolasco, P. Brandão, R. A. S. Ferreira, R. S. Carvalho, M. Cremona and L. D. Carlos, Eur. J. Inorg. Chem., 2020, DOI: 10.1002/ejic.202000027.

3 (a) A. N. Al-Khalili, I. J. Al-Busaidi, R. Ilmi, M. Al-Mandhary, M. S. Khan and N. K. Al-Rasbi, Inorg. Chim. Acta, 2020, 501, 119226; (b) Y. Liu, J. Ma, C. Xu, Y. Yang, M. Xia, H. Jiang and W. Liu, Dalton Trans., 2018, 47, 13543-13549; (c) W. Liu, X. Huang, C. Chen, C. Xu, J. Ma, L. Yang, W. Wang, W. Dou and W. Liu, Chem. - Eur. J., 2019, 25, 1090-1097.

4 L. Zhou, L. Li, Y. Jiang, R. Cui, Y. Li, X. Zhao and H. Zhang, ACS Appl. Mater. Interfaces, 2015, 7, 16046-16053.

5 (a) P. K. Shahi, A. K. Singh, S. K. Singh, S. B. Rai and B. Ullrich, ACS Appl. Mater. Interfaces, 2015, 7, 18231-18239; (b) K. Nakamura, Y. Hasegawa, H. Kawai, N. Yasuda, N. Kanehisa, Y. Kai, T. Nagamura, S. Yanagida and Y. Wada, J. Phys. Chem. A, 2007, 111, 3029-3037; (c) R. Ilmi, S. Anjum, A. Haque and M. S. Khan, J. Photochem. Photobiol., A, 2019, 383, 111968; (d) K. Nakamura, Y. Hasegawa, H. Kawai, N. Yasuda, Y. Wada and S. Yanagida, J. Alloys Compd., 2006, 408-412, 771-775.

6 R. Ilmi, A. Haque, I. J. Al-Busaidi, N. K. Al Rasbi and M. S. Khan, Dyes Pigm., 2019, 162, 59-66.

7 (a) O. Moudam, B. C. Rowan, M. Alamiry, P. Richardson, B. S. Richards, A. C. Jones and N. Robertson, Chem. Commun., 2009, 6649-6651, DOI: 10.1039/B914978C; (b) A. S. Kalyakina, V. V. Utochnikova, M. Zimmer, F. Dietrich, A. M. Kaczmarek, R. Van Deun, A. A. Vashchenko, A. S. Goloveshkin, M. Nieger, M. Gerhards, U. Schepers and S. Brase, Chem. Commun., 2018, 54, 5221-5224.

8 O. Laporte and W. F. Meggers, J. Opt. Soc. Am., 1925, 11, 459-463. 9 (a) N. Sabbatini, M. Guardigli and I. Manet, in Handbook on the Physics and Chemistry of Rare Earths, ed. K. A. Gschneidner Jr. and E. LeRoy, Elsevier, 1996, vol. 23, pp. 69-119; (b) S. I. Weissman, J. Chem. Phys., 1942, 10, 214-217.

10 (a) K. Binnemans, in Handbook on the Physics and Chemistry of Rare Earths, ed. J. C. B. K. A. Gschneidner and V. K. Pecharsky, Elsevier, 2005, vol. 35, pp. 107-272; (b) R. Ilmi, S. Kansız, N. K. Al Rasbi, N. Dege, P. R. Raithby and M. S. Khan, New J. Chem., 2020, DOI: 10.1039/c9nj06287d; (c) R. Ilmi and K. Iftikhar, Polyhedron, 2017, 127, 191-202.

11 (a) E. Busby, J. Xia, Q. Wu, J. Z. Low, R. Song, J. R. Miller, X. Y. Zhu, L. M. Campos and M. Y. Sfeir, Nat. Mater., 2015, 
14, 426; (b) Q. Zhang, B. Li, S. Huang, H. Nomura, H. Tanaka and C. Adachi, Nat. Photonics, 2014, 8, 326.

12 T. Koizuka, M. Yamamoto, Y. Kitagawa, T. Nakanishi, K. Fushimi and Y. Hasegawa, Bull. Chem. Soc. Jpn., 2017, 90, 1287-1292.

13 (a) J. Kido, K. Nagai and Y. Ohashi, Chem. Lett., 1990, 657-660; (b) J. Kido, K. Nagai, Y. Okamoto and T. Skotheim, Chem. Lett., 1991, 1267-1270; (c) J. Kido, K. Nagai and Y. Okamoto, J. Alloys Compd., 1993, 192, 30-33.

14 H. Xu, L.-H. Wang, X.-H. Zhu, K. Yin, G.-Y. Zhong, X.-Y. Hou and W. Huang, J. Phys. Chem. B, 2006, 110, 3023-3029.

15 H. Xu, K. Yin and W. Huang, Chem. - Eur. J., 2007, 13, 10281-10293.

16 B. Zhao, H. Zhang, Y. Miao, Z. Wang, L. Gao, H. Wang, Y. Hao, B. Xu and W. Li, J. Mater. Chem. C, 2017, 5, 12182-12188.

17 R. Ilmi, S. Kansiz, N. Dege and M. S. Khan, J. Photochem. Photobiol., A, 2019, 377, 268-281.

18 M. A. Filho, J. D. L. Dutra, H. L. Cavalcanti, G. B. Rocha, A. M. Simas and R. O. Freire, J. Chem. Theory Comput., 2014, 10, 3031-3037.

19 J. J. P. Stewart, MOPAC2016, Stewart Computational Chemistry, CO, Colorado Springs, USA, 2016.

20 C. R. Groom, I. J. Bruno, M. P. Lightfoot and S. C. Ward, Acta Crystallogr., Sect. B: Struct. Sci., Cryst. Eng. Mater., 2016, 72, 171-179.

21 F. Neese, Wiley Interdiscip. Rev.: Comput. Mol. Sci., 2018, 8, e1327.

22 M. Dolg, H. Stoll and H. Preuss, J. Chem. Phys., 1989, 90, 1730-1734.

23 S. M. Ali, S. Pahan, A. Bhattacharyya and P. K. Mohapatra, Phys. Chem. Chem. Phys., 2016, 18, 9816-9828.

24 (a) O. L. Malta, J. Lumin., 1997, 71, 229-236; (b) F. R. G. e Silva and O. L. Malta, J. Alloys Compd., 1997, 250, 427-430.

25 W. T. Carnall, H. Crosswhite and H. M. Crosswhite, Energy level structure and transition probabilities in the spectra of the trivalent lanthanides in $\mathrm{LaF}_{3}$, United States, 1978.

26 J. D. Dutra, T. D. Bispo and R. O. Freire, J. Comput. Chem., 2014, 35, 772-775.

27 A. N. Carneiro Neto, E. E. S. Teotonio, G. F. de Sá, H. F. Brito, J. Legendziewicz, L. D. Carlos, M. C. F. C. Felinto,
P. Gawryszewska, R. T. Moura, R. L. Longo, W. M. Faustino and O. L. Malta, in Handbook on the Physics and Chemistry of Rare Earths, ed. J.-C. G. Bünzli and V. K. Pecharsky, Elsevier, 2019, vol. 56, pp. 55-162.

28 (a) K. L. Nash, R. D. Rogers, J. Ferraro and J. Zhang, Inorg. Chim. Acta, 1998, 269, 211-223; (b) U. Englert, B. Ganter, T. Wagner and W. Kläui, Z. Anorg. Allg. Chem., 1998, 624, 970-974.

29 F. Nüesch, D. Berner, E. Tutiš, M. Schaer, C. Ma, X. Wang, B. Zhang and L. Zuppiroli, Adv. Funct. Mater., 2005, 15, 323-330.

30 K. Miyata, T. Nakagawa, R. Kawakami, Y. Kita, K. Sugimoto, T. Nakashima, T. Harada, T. Kawai and Y. Hasegawa, Chem. - Eur. J., 2011, 17, 521-528.

31 R. Ilmi, A. Haque and M. S. Khan, J. Photochem. Photobiol., A, 2019, 370, 135-144.

32 J. D. L. Dutra, N. B. D. Lima, R. O. Freire and A. M. Simas, Sci. Rep., 2015, 5, 13695.

33 M. Latva, H. Takalo, V. M. Mukkala, C. Matachescu, J. C. RodriguezUbis and J. Kankare, J. Lumin., 1997, 75, 149-169.

34 M. Congiu, M. Alamiry, O. Moudam, S. Ciorba, P. R. Richardson, L. Maron, A. C. Jones, B. S. Richards and N. Robertson, Dalton Trans., 2013, 42, 13537-13545.

35 O. L. Malta, F. R. G. E. Silva and R. Longo, Chem. Phys. Lett., 1999, 307, 518-526.

36 (a) P.-P. Sun, J.-P. Duan, H.-T. Shih and C.-H. Cheng, Appl. Phys. Lett., 2002, 81, 792-794; (b) F. Liang, Q. Zhou, Y. Cheng, L. Wang, D. Ma, X. Jing and F. Wang, Chem. Mater., 2003, 15, 1935-1937.

37 H. Xu, K. Yin and W. Huang, J. Phys. Chem. C, 2010, 114, 1674-1683.

38 (a) S. Zhang, G. A. Turnbull and I. D. W. Samuel, Org. Electron., 2012, 13, 3091-3096; (b) Y. Liu, J. Li, C. Li, J. Song, Y. Zhang, J. Peng, X. Wang, M. Zhu, Y. Cao and W. Zhu, Chem. Phys. Lett., 2007, 433, 331-334; (c) M. Pietraszkiewicz, M. Maciejczyk, I. D. W. Samuel and S. Zhang, J. Mater. Chem. C, 2013, 1, 8028-8032; (d) S. Wang, J. Zhang, Y. Hou, C. Du and Y. Wu, J. Mater. Chem., 2011, 21, 7559-7561; (e) Y. Liu, Y. Wang, H. Guo, M. Zhu, C. Li, J. Peng, W. Zhu and Y. Cao, J. Phys. Chem. C, 2011, 115, 4209-4216. 